\title{
Search for new physics in the multijet and missing transverse momentum final state in proton-proton collisions at $\sqrt{s}=8 \mathrm{TeV}$
}

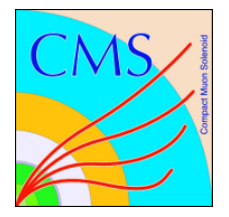

\section{The CMS collaboration}

E-mail: cms-publication-committee-chair@cern.ch

ABSTRACT: A search for new physics is performed in multijet events with large missing transverse momentum produced in proton-proton collisions at $\sqrt{s}=8 \mathrm{TeV}$ using a data sample corresponding to an integrated luminosity of $19.5 \mathrm{fb}^{-1}$ collected with the CMS detector at the LHC. The data sample is divided into three jet multiplicity categories (3-5, 6-7, and $\geq 8$ jets), and studied further in bins of two variables: the scalar sum of jet transverse momenta and the missing transverse momentum. The observed numbers of events in various categories are consistent with backgrounds expected from standard model processes. Exclusion limits are presented for several simplified supersymmetric models of squark or gluino pair production.

Keywords: Supersymmetry, Hadron-Hadron Scattering

ARXIV EPRINT: 1402.4770 


\section{Contents}

1 Introduction 1

2 The CMS detector and event reconstruction 2

3 Sample selection $\quad 3$

4 Background estimation $\quad 4$

4.1 Estimation of $\mathrm{Z}(\nu \bar{\nu})+$ jets background 5

4.2 Estimation of the lost-lepton background 6

4.3 Estimation of the hadronic $\tau$ lepton background 8

4.4 Estimation of the QCD multijet background 9

5 Results and interpretation $\quad 10$

$\begin{array}{lll}6 & \text { Summary } & 15\end{array}$

$\begin{array}{lr}\text { The CMS collaboration } & 20\end{array}$

\section{Introduction}

The standard model of particle physics (SM) successfully describes a wide variety of observations in high energy physics. The recent discovery of a new scalar boson with a mass of about $125 \mathrm{GeV}$ [1-3] at the CERN Large Hadron Collider (LHC) marks another success for the SM, as its properties measured so far are consistent with those of the long-sought Higgs boson. However, its mass is predicted to be unstable against quadratically divergent quantum-loop corrections, which suggests the presence of physics beyond the SM. Supersymmetry (SUSY) is a well-explored extension that addresses various shortcomings of the SM. SUSY postulates a new symmetry, relating fermionic and bosonic degrees of freedom, and introduces a superpartner for each SM particle. Radiative corrections due to SUSY particles can compensate the contribution of the SM particles and thereby stabilize the mass of the Higgs boson. In $R$-parity-conserving models [4], SUSY particles are produced in pairs, and the lightest SUSY particle (LSP) is stable. If weakly interacting and neutral, the LSP is a potential dark matter candidate.

This paper reports an inclusive search for physics beyond the SM in multijet events with large missing transverse momentum produced in pp collisions at a centre-of-mass energy $\sqrt{s}=8 \mathrm{TeV}$ at the LHC. The data sample used corresponds to an integrated luminosity of $19.5 \mathrm{fb}^{-1}$ collected by the Compact Muon Solenoid (CMS) experiment [5]. This final state is motivated by many extensions of the SM, for example those given in refs. [6-8]. At the LHC, both the CMS and ATLAS collaborations have performed SUSY 
searches in all-hadronic final states [9-17]. For all these searches, the observed numbers of events were consistent with the expected SM background, and exclusion limits were set in the context of the constrained minimal supersymmetric extension of the standard model (CMSSM) [18-20] and various simplified models [21, 22]. Contrary to the CMSSM case, the masses of particles are free parameters in simplified models, thus allowing a generic study of the parameter space of SUSY and SUSY-like theories. Simplified models of squark and gluino pair production are used to interpret the search results in this paper.

This analysis follows previous inclusive searches $[9,10]$ that require at least three jets in the final state. These searches are most sensitive to the hypothetical production of pairs of squarks and gluinos, where the squarks (gluinos) each decay to one (two) jets and an undetected LSP. We extend the analyses of refs. $[9,10]$ by subdividing the data into three exclusive jet multiplicity categories: $N_{\text {Jets }}=3-5,6-7$, and $\geq 8$, which renders the analysis more sensitive to a variety of final-state topologies resulting from longer cascades of squarks and gluinos, and hence in a larger number of jets. The search regions with higher jet multiplicities extend the sensitivity of the analysis to models in which the gluino often decays into top quarks. While other analyses exploit the presence of bottom-quark jets in signal events to discriminate against background $[12,13]$, this analysis follows a complementary strategy by requiring a large number of jets, thus helping to keep the signal efficiency for fully hadronic final states as high as possible.

The events in each jet multiplicity category are further divided according to variables that characterize the total visible hadronic activity $\left(H_{\mathrm{T}}\right)$ and the momentum imbalance $\left(H_{\mathrm{T}}\right)$ in an event, both defined in the plane transverse to the beam. Due to the presence of a number of energetic jets and two LSPs in the final state, the signal events are expected to have large $H_{\mathrm{T}}$ and $H_{\mathrm{T}}$. The main SM processes contributing to this final state are $\mathrm{Z}+$ jets events, where the $\mathrm{Z}$ boson decays to a pair of neutrinos $(\mathrm{Z}(\nu \bar{\nu})+$ jets $)$, and $\mathrm{W}+$ jets and $\mathrm{t} \overline{\mathrm{t}}$ events, where a $\mathrm{W}$ boson decays to an e, $\mu$, or $\tau$ lepton $(\mathrm{W}(\ell \nu)+$ jets). The presence of at least one neutrino in these events provides a source of genuine $\not_{\mathrm{T}}$. Another background category is quantum chromodynamics (QCD) multijet events with large $H_{\mathrm{T}}$ from leptonic decays of heavy-flavour hadrons inside the jets, jet energy mismeasurement, or instrumental noise and non-functioning detector components. All these backgrounds are determined using the data, with as little reliance on simulation as possible.

\section{The CMS detector and event reconstruction}

The CMS detector is a multipurpose apparatus, described in detail in ref. [5]. The CMS coordinate system is defined with the origin at the centre of the detector and the $z$ axis along the anticlockwise beam direction. The polar angle $\theta$ is measured with respect to the $z$ axis, and the azimuthal angle $\phi$ (measured in radians) in the plane perpendicular to that axis. Charged-particle trajectories are measured with a silicon pixel and strip tracker, covering $|\eta|<2.5$, where the pseudorapidity $\eta$ is defined as $\eta=-\ln [\tan (\theta / 2)]$. Immersed in the $3.8 \mathrm{~T}$ magnetic field provided by a $6 \mathrm{~m}$ diameter superconducting solenoid, which also encircles the calorimeters, the tracking system provides transverse momentum $\left(p_{\mathrm{T}}\right)$ resolution of approximately $1.5 \%$ for charged particles with $p_{\mathrm{T}} \sim 100 \mathrm{GeV}$. A lead-tungstate 
crystal electromagnetic calorimeter and a brass-and-scintillator hadron calorimeter surround the tracking volume and cover the region $|\eta|<3$. Steel and quartz-fibre hadron forward calorimeters extend the coverage to $|\eta| \leq 5$. Muons are identified in gas ionization detectors embedded in the steel flux return yoke of the magnet. The events used for this search are recorded using a two-level trigger system described in ref. [5].

The recorded events are required to have at least one well-identified interaction vertex with $z$ position within $24 \mathrm{~cm}$ from the nominal centre of the detector and transverse distance from the $z$ axis less than $2 \mathrm{~cm}$. The primary vertex is the one with the largest sum of $p_{\mathrm{T}}$-squared of all the associated tracks, and is assumed to correspond to the hardscattering process. The events are reconstructed using a particle-flow (PF) algorithm [23]. This algorithm reconstructs a list of particles in each event, namely charged and neutral hadrons, photons, muons, and electrons, combining the information from the tracker, the calorimeters, and the muon system. These particles are then clustered into jets using the anti- $k_{\mathrm{T}}$ clustering algorithm [24] with a size parameter of 0.5. Contributions from additional pp collisions overlapping with the event of interest (pileup) are mitigated by discarding charged particles not associated with the primary vertex and using the Fastjet tools $[25,26]$ to account for the neutral pileup component. Corrections to jet energy are applied to account for the variation of the response in $p_{\mathrm{T}}$ and $\eta$ [27]. Missing transverse momentum $\left(\mathscr{E}_{\mathrm{T}}\right)$ is reconstructed as magnitude of the vector sum of $p_{\mathrm{T}}$ of all the reconstructed PF particles [28, 29].

\section{Sample selection}

The search regions are first defined using a loose baseline selection with the following requirements:

- $N_{\text {Jets }} \geq 3$, where $N_{\text {Jets }}$ is the number of jets with $p_{\mathrm{T}}>50 \mathrm{GeV}$ and $|\eta|<2.5$.

- $H_{\mathrm{T}}>500 \mathrm{GeV}$, with $H_{\mathrm{T}}=\sum_{\text {jets }} p_{\mathrm{T}}$, where the sum includes all jets with $p_{\mathrm{T}}>50 \mathrm{GeV}$ and $|\eta|<2.5$.

- $H_{\mathrm{T}}>200 \mathrm{GeV}$, with $H_{\mathrm{T}}=\left|\vec{H}_{\mathrm{T}}\right|=\left|-\sum_{\text {jets }} \vec{p}_{\mathrm{T}}\right|$, where in this case, jets are required to satisfy $p_{\mathrm{T}}>30 \mathrm{GeV}$ and $|\eta|<5$.

- $\left|\Delta \phi\left(\vec{p}_{\mathrm{T}}^{\mathrm{Jet} 1}, \vec{H}_{\mathrm{T}}\right)\right|>0.5,\left|\Delta \phi\left(\vec{p}_{\mathrm{T}}^{\text {Jet2}}, \vec{H}_{\mathrm{T}}\right)\right|>0.5$, and $\left|\Delta \phi\left(\vec{p}_{\mathrm{T}}^{\text {Jet3 }, \vec{H}_{\mathrm{T}}}\right)\right|>0.3$, vetoing the events where $\vec{H}_{\mathrm{T}}$ is aligned with one of the three highest $p_{\mathrm{T}}$ jets. This requirement rejects most of the QCD multijet events in which a single mismeasured jet yields high $H_{\mathrm{T}}$.

- Events containing isolated muons or electrons with $p_{\mathrm{T}}>10 \mathrm{GeV}$ are vetoed in order to reject $t \bar{t}$ and $\mathrm{W} / \mathrm{Z}+$ jets events with leptons in the final state. Both the e and $\mu$ are required to produce a good quality track that is matched to the primary interaction vertex [30,31]. The isolation is measured as the scalar $p_{\mathrm{T}}$ sum of PF particles $\left(p_{\mathrm{T}}^{\mathrm{sum}}\right)$, except the lepton itself, within a cone of width $\Delta R=\sqrt{(\Delta \eta)^{2}+(\Delta \phi)^{2}}=0.3$ for e ( 0.4 for $\mu$ ) around the lepton. The $p_{\mathrm{T}}^{\text {sum }}$ is required to be less than $20 \%(15 \%)$ of the $p_{\mathrm{T}}$ of the e $(\mu)$. 
- In addition, events affected by instrumental effects, particles from non-collision sources, or poorly reconstructed kinematic variables are rejected (event cleaning) $[28,29]$. Events are also rejected if a jet with $p_{\mathrm{T}}>30 \mathrm{GeV}$ has more than 95\% of its energy from PF photon candidates or more than $90 \%$ from PF neutral hadron candidates.

The data sample used for this analysis was collected using trigger algorithms that required events to have $H_{\mathrm{T}}>350 \mathrm{GeV}$ and $\mathbb{E}_{\mathrm{T}}>100 \mathrm{GeV}$. The trigger efficiencies are measured to be greater than $99 \%$ for the offline baseline selection of $H_{\mathrm{T}}>500 \mathrm{GeV}$ and $H_{\mathrm{T}}>200 \mathrm{GeV}$ in all jet multiplicity categories used in this search. A sample of 11753 events is selected after applying the baseline criteria. The selected events are divided into 36 non-overlapping search regions defined in terms of $N_{\text {Jets }}, H_{\mathrm{T}}$, and $\not H_{\mathrm{T}}$, as listed in the first three columns of table 1 .

Several Monte Carlo (MC) simulation samples are used to model the signal as well as to develop and validate the background estimation methods. The t $\bar{t}, \mathrm{~W} / \mathrm{Z}+\mathrm{jets}, \gamma+$ jets, and QCD multijet background samples are produced using the MADGRAPH5 [32] generator at leading order (LO), interfaced with the PYTHIA 6.4.24 [33] parton-shower model, and scaled to the next-to-leading order (NLO) or next-to-next-to-leading order cross section predictions $[34,35]$. The events are processed through a GEANT4 simulation of the detector [36]. The SUSY signal samples are generated using MAdGraph5, the CTEQ6L [37] parton distribution functions (PDF), and are simulated using the CMS fast simulation package [38]. The underlying event description used for the MC simulated samples is described in ref. [39]. The effect of pileup interactions is included by adding a number of simulated minimum bias events, on top of the hard interaction, to match the distribution observed in data.

\section{Background estimation}

In this search, all backgrounds are measured from data using methods similar to those described in refs. $[9,10]$. The $\mathrm{Z}(\nu \bar{\nu})+$ jets background is estimated using $\gamma+$ jets events, exploiting their electroweak correspondence to $\mathrm{Z}+$ jets production for boson $p_{\mathrm{T}}$ above $\sim 100 \mathrm{GeV}$. The $\mathrm{Z}+$ jets and $\gamma+$ jets events exhibit similar characteristics, apart from electroweak coupling differences and asymptotically vanishing residual mass effects. The $\mathrm{t} \overline{\mathrm{t}}$ or $\mathrm{W}(\ell \nu)+$ jets events satisfy the search selection when the $\mathrm{e} / \mu$ is not identified or isolated, or is out of the detector acceptance ("lost-lepton" background) or when a $\tau$ lepton decays hadronically ( $\tau_{\mathrm{h}}$ background). The lost-lepton background is estimated by reweighting events in a $\mu+$ jets data control sample with measured lepton efficiencies. The estimation of the $\tau_{\mathrm{h}}$ background starts from a similar $\mu+$ jets sample, replacing the muon with a jet sampled as a function of jet $p_{\mathrm{T}}$ from $\tau_{\mathrm{h}}$ templates obtained from simulation. The QCD multijet background is measured using a "rebalance-and-smear" method $[9,10]$. The kinematical characteristics of multijet events are predicted from data by applying a fitting procedure that imposes zero missing transverse momentum on each event, and then smearing the jets according to data-corrected jet energy resolution values. The relative contribution of the various backgrounds varies in the different search regions. 


\subsection{Estimation of $\mathrm{Z}(\nu \bar{\nu})+$ jets background}

Photons and $\mathrm{Z}$ bosons exhibit similar kinematic properties at high $p_{\mathrm{T}}$, and therefore the hadronic component of an event containing either a high- $p_{\mathrm{T}}$ photon or $\mathrm{Z}$ boson is similar [40-43]. The $\gamma+$ jets sample used to evaluate the $\mathrm{Z}(\nu \bar{\nu})+$ jets event rate is collected by triggering on events with a $\gamma$ candidate and large $H_{\mathrm{T}}$. The photon candidates are reconstructed using the energy deposited in the electromagnetic calorimeter [44, 45]. Photon candidates with $p_{\mathrm{T}}>100 \mathrm{GeV}$ and $|\eta|<1.44$ or $1.566<|\eta|<2.5$ are used in this analysis, and are required to have their lateral shower profile consistent with that of a photon produced in the hard-scattering process (a prompt photon). To veto electrons misidentified as photons, the candidates with an associated track in the pixel detector are rejected. A photon candidate is required to satisfy tight isolation requirements based on the sum over $p_{\mathrm{T}}$ values of the $\mathrm{PF}$ candidates that lie within a cone of radius $\Delta R=0.3$ around the direction of its momentum.

The contribution to the $\gamma+$ jets control sample from events in which the photon candidate originates from the misidentification of jet fragments (background photons) is measured using a template method, which exploits the difference between the shower profile of prompt (signal) and background photons, using the distribution of a modified second moment of the electromagnetic energy cluster around its mean $\eta$ position [44]. The distribution (template) for background events is obtained from a sideband region defined by selecting photons that satisfy very loose photon identification and isolation requirements but fail the stringent isolation requirements. The distribution for signal events is obtained from simulation. The sum of the two templates is fit to the observed distribution, with the normalization (background and signal yields) of each template determined in the fit. On average, $93 \%$ of selected $\gamma+$ jets candidate events are determined to originate from prompt photons.

To mimic the missing momentum due to the neutrinos from the decay of the $\mathrm{Z}$ boson, the photon candidate is not included in the calculation of $H_{\mathrm{T}}$ and $H_{\mathrm{T}}$ for the $\gamma+$ jets events. The number of $\mathrm{Z}(\nu \bar{\nu})+$ jets events is then estimated by correcting the number of $\gamma+$ jets events for photon acceptance and reconstruction efficiency, and scaling the result with the ratio relating the production cross section of the two processes $\left(R_{\mathrm{Z} / \gamma}\right)$ in the various search regions. Therefore, the ratio $R_{\mathrm{Z} / \gamma}$, which we derive from simulation, is studied as a function of $H_{\mathrm{T}}, H_{\mathrm{T}}$, and $N_{\text {Jets }}$ using events generated with MADGRAPH (up to four partons) that are processed through the PYTHIA parton shower algorithm to generate additional jets. The ratio exhibits a strong dependence on $\not_{\mathrm{T}}$ for values below around $500 \mathrm{GeV}$ (figure 1 (a)), but changes by only $(12 \pm 5) \%$ as $H_{\mathrm{T}}$ varies between 500 and $1500 \mathrm{GeV}$ (figure $1(\mathrm{~b})$ ), which is the region of interest to this search. The ratio is parametrized as a linear function of $N_{\text {Jets }}$ in several $H_{\mathrm{T}}$ ranges, $200<H_{\mathrm{T}}<300 \mathrm{GeV}, 300<H_{\mathrm{T}}<450 \mathrm{GeV}$, and $\not_{\mathrm{T}}>450 \mathrm{GeV}$, as shown in figure $1(\mathrm{c})$. The predicted numbers of $\mathrm{Z}(\nu \bar{\nu})+$ jets events and uncertainties for various search regions are summarized in table 1.

The theoretical uncertainty associated with $R_{\mathrm{Z} / \gamma}$ is estimated using $\mathrm{Z}\left(\mu^{+} \mu^{-}\right)+$jets events selected from data and simulation, by requiring two opposite-sign muons to satisfy the muon selection and to form an invariant mass within $\pm 20 \mathrm{GeV}$ of the $\mathrm{Z}$ boson mass. The 

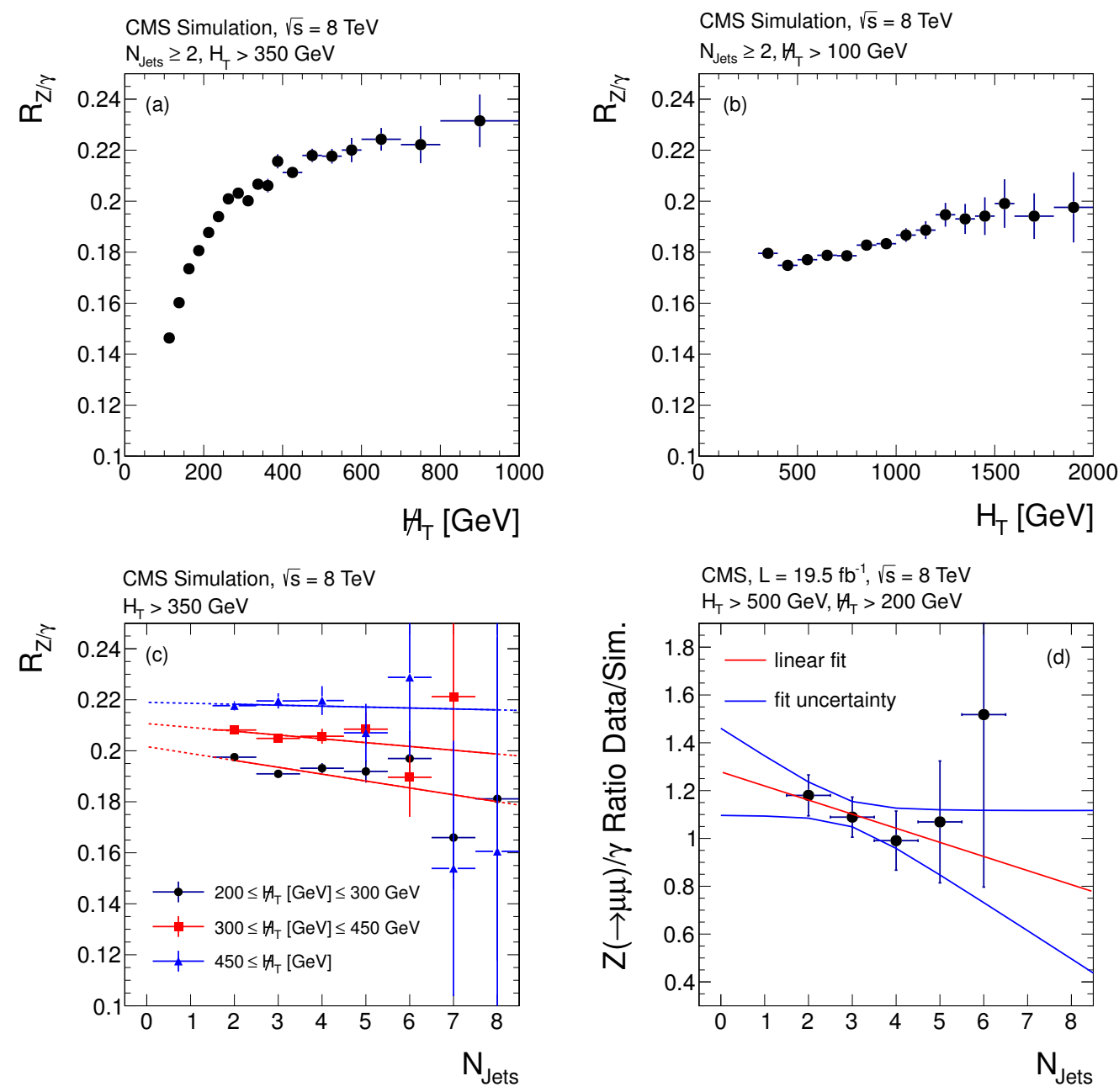

Figure 1. The simulated ratio $R_{\mathrm{Z} / \gamma}$ as a function of (a) $H_{\mathrm{T}}$, (b) $H_{\mathrm{T}}$, (c) $N_{\mathrm{Jets}}$, where the values for three $H_{\mathrm{T}}$ bins are shown with linear fits, and (d) the double ratio of $R_{\mathrm{Z}\left(\mu^{+} \mu^{-}\right) / \gamma}$, using events from data to those from simulation; the linear fit and its uncertainty band are overlaid.

double ratio of $R_{\mathrm{Z}\left(\mu^{+} \mu^{-}\right) / \gamma}$ using events from data to those from simulation is parametrized as a function of $N_{\text {Jets }}$ using a linear function, as shown in figure $1(\mathrm{~d})$, and is used to correct $R_{\mathrm{Z} / \gamma}$ for a given jet multiplicity. The fitting procedure results in uncertainties of $20 \%$, $25 \%$, and $45 \%$ for the background predicted in the search regions with $N_{\text {Jets }}=3-5,6-7$, and $\geq 8$, respectively. The difference in the modeling of photon identification and isolation in the simulation and data leads to uncertainties of $2-5 \%, 10-20 \%$, and $20-25 \%$ on the estimated number of $\mathrm{Z}(\nu \bar{\nu})+$ jets events for the three jet multiplicity intervals, respectively. The subtraction of events with non-prompt photons from QCD multijet events amounts to less than a $5 \%$ uncertainty for the final background prediction.

\subsection{Estimation of the lost-lepton background}

The lost-lepton background is estimated from a $\mu+$ jets control sample, selected with the same criteria as used for the search, except that events are required to have exactly one well- 

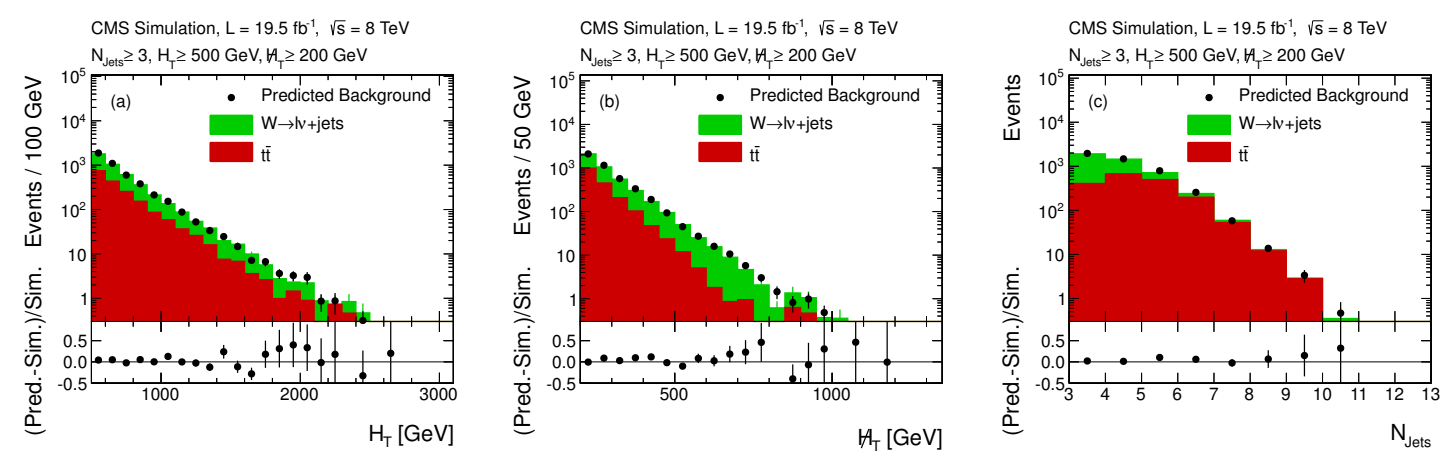

Figure 2. Predicted (a) $H_{\mathrm{T}}$, (b) $H_{\mathrm{T}}$, and (c) $N_{\text {Jets }}$ distributions found from applying the lost-lepton background evaluation method to simulated t $\overline{\mathrm{t}}$ and $\mathrm{W}+$ jets events (solid points) in comparison to the genuine $t \bar{t}$ and $\mathrm{W}+$ jets background from simulation (shaded curves). Only statistical uncertainties are shown.

reconstructed and isolated $\mu$ with $p_{\mathrm{T}}^{\mu}>10 \mathrm{GeV}$. The events are collected with the same trigger that is used to search for the signal. The transverse mass $m_{\mathrm{T}}=\sqrt{2 p_{\mathrm{T}}^{\mu} \mathbb{E}_{\mathrm{T}}[1-\cos (\Delta \phi)]}$ is required to be less than $100 \mathrm{GeV}$ in order to select events containing $\mathrm{W} \rightarrow \mu \nu$ decays as well as to reject possible signal events. Here $\Delta \phi$ is the azimuthal angle between the $\vec{p}_{\mathrm{T}}^{\mu}$ and the $\overrightarrow{E_{\mathrm{T}}}$ directions.

Using the reconstruction and isolation efficiencies $\epsilon_{\text {reco }}^{\mathrm{e}, \mu}$ and $\epsilon_{\text {iso }}^{\mathrm{e}, \mu}$ of the electrons and muons, the events in the isolated muon control sample are weighted by $\left(1 / \epsilon_{\text {iso }}^{\mu}\right) \times$ $\left[\left(1-\epsilon_{\text {reco }}^{\mathrm{e}, \mu}\right) / \epsilon_{\text {reco }}^{\mu}\right]$ in order to estimate the number of events with unidentified leptons, and by $\left(\epsilon_{\text {reco }}^{\mathrm{e}, \mu} / \epsilon_{\text {reco }}^{\mu}\right) \times\left[\left(1-\epsilon_{\text {iso }}^{\mathrm{e}, \mu}\right) / \epsilon_{\text {iso }}^{\mu}\right]$ to estimate the number of events with non-isolated leptons in the signal region. The predicted number of lost-lepton events is corrected to account for the detector and kinematic acceptance of the muons. The lepton efficiencies and kinematic acceptance factors are obtained from the MC simulation of $\mathrm{W}+$ jets and $\mathrm{t} \overline{\mathrm{t}}$ events and are determined in bins of $N_{\mathrm{Jets}}, H_{\mathrm{T}}$, and $H_{\mathrm{T}}$.

This method is validated using simulated $t \bar{t}$ and $\mathrm{W}+$ jets events. The single-muon events selected from the simulated samples are used to predict the number of background events expected in the zero-lepton search regions. The resulting $H_{\mathrm{T}}, H_{\mathrm{T}}$, and $N_{\text {Jets }}$ distributions are compared in figure 2 to the genuine ones obtained from $\mathrm{t} \overline{\mathrm{t}}$ and $\mathrm{W}+\mathrm{jets}$ events simulated at the detector level. The predicted distributions closely resemble the genuine ones.

The number of lost-lepton events predicted from data using the method described above, and the corresponding uncertainties, are listed in table 1 for each search region. The dominant uncertainties arise from the limited number of single-muon events in most of the search regions. The differences in lepton reconstruction and isolation efficiencies between data and MC simulation are evaluated using a "tag-and-probe" method [46] on $\mathrm{Z}\left(\mu^{+} \mu^{-}\right)+$jets events. The lepton reconstruction and isolation efficiencies are measured in bins of lepton $p_{\mathrm{T}}$ and $\Delta R$ relative to the closest jet. This method renders these efficiencies insensitive to the kinematic differences between $\mathrm{Z}\left(\ell^{+} \ell^{-}\right)+$jets events and $\mathrm{t} \overline{\mathrm{t}}$ and $\mathrm{W}+$ jets events. Relative differences between the predictions using efficiencies extracted from data and MC simulation result in $10-25 \%, 10-30 \%$, and $15-24 \%$ uncertainties for the predicted 
background for various $H_{\mathrm{T}}$ and $H_{\mathrm{T}}$ search bins with $N_{\text {Jets }}=3-5,6-7$, and $\geq 8$, respectively. An additional uncertainty of $15 \%$ for $N_{\text {Jets }}=3-5$ and $40 \%$ for $N_{\text {Jets }} \geq 6$ is assigned based on the statistical precision of the validation of this background estimation method. Variation of the PDFs following the procedure of ref. [47] affects the muon acceptance, and leads to an uncertainty of less than $4 \%$ on the final prediction. Any mismodeling of anomalous $\mathbb{E}_{\mathrm{T}}[28]$ affects the simulated $m_{\mathrm{T}}$ and results in $3 \%$ uncertainty for the predicted lost-lepton background.

\subsection{Estimation of the hadronic $\tau$ lepton background}

The $\tau_{\mathrm{h}}$ background is estimated from a sample of $\mu+$ jets events, selected with an inclusive single $\mu$ or $\mu+\geq 2$-jet trigger, by requiring exactly one $\mu$ with $p_{\mathrm{T}}>20 \mathrm{GeV}$ and $|\eta|<2.1$. As in the estimation of the lost-lepton background, only events with $m_{\mathrm{T}}<100 \mathrm{GeV}$ are considered. The $\mu+$ jets and $\tau_{\mathrm{h}}+$ jets events arise from the same physics processes; hence the hadronic component of the two samples is the same aside from the response of the detector to a muon or a $\tau_{\mathrm{h}}$ jet. To account for this difference, the muon is replaced by a simulated $\tau_{\mathrm{h}}$ jet, whose $p_{\mathrm{T}}$ value is randomly sampled from an $\mathrm{MC}$ response function, $p_{\mathrm{T}}^{\mathrm{Jet}} / p_{\mathrm{T}}^{\tau}$. Here, the $p_{\mathrm{T}}^{\tau}$ is the transverse momentum of a generated hadronically decaying $\tau$ lepton selected from simulated $\mathrm{t} \overline{\mathrm{t}}$ and $\mathrm{W}(\tau \nu)+$ jets events and $p_{\mathrm{T}}^{\mathrm{Jet}}$ is that of a reconstructed jet matching the $\tau$ lepton in $\eta-\phi$ space. In order to sample the response function completely, this procedure is repeated one hundred times for each event. The $N_{\mathrm{Jets}}, H_{\mathrm{T}}$, and $\not_{\mathrm{T}}$ values of the events are recalculated, now including this $\tau_{\mathrm{h}}$ jet, and search region selection criteria are applied to predict the $\tau_{\mathrm{h}}$ background. The predicted background is corrected for the trigger efficiency, muon selection efficiency, kinematic and detector acceptance, and the ratio of branching fractions $\mathcal{B}\left(\mathrm{W} \rightarrow \tau_{\mathrm{h}} \nu\right) / \mathcal{B}(\mathrm{W} \rightarrow \mu \nu)=0.6476 \pm 0.0024$ [48]. The muon isolation and reconstruction efficiencies are obtained from $\mathrm{MC}$ simulation of $\mathrm{W}+$ jets and $\mathrm{t} \overline{\mathrm{t}}$ events in bins of lepton $p_{\mathrm{T}}$ and $\Delta R$ relative to the closest jet. To account for the difference in efficiencies measured in data and MC simulation, the predicted numbers of $\tau_{\mathrm{h}}+$ jets events are corrected by $4.9 \%, 4.7 \%$, and $3.5 \%$ for $N_{\text {Jets }}=3-5,6-7$, and $\geq 8$, respectively. The predicted $\tau_{\mathrm{h}}$ background and uncertainties are shown in table 1 for all the search regions.

The $\tau_{\mathrm{h}}$ background estimation method is validated by applying it to simulated $\mathrm{W}+$ jets and $\mathrm{t} \overline{\mathrm{t}} \mathrm{MC}$ samples. The results are shown in figure 3 in comparison to the genuine $\tau_{\mathrm{h}}$ background from the simulated events. To evaluate the performance of the method for events with varying hadronic activity, the method is validated in each search bin. Uncertainties of $10 \%, 20 \%$, and $20 \%$ are assigned to the predicted rates for events with $N_{\text {Jets }}=3-5,6-7$, and $\geq 8$ respectively, mainly to reflect the level of statistical precision for this validation. Due to the multiple sampling of the response template, the statistical uncertainty of the prediction is evaluated with a set of pseudo-experiments using a bootstrap technique [49]. Relative differences between the predictions using efficiencies extracted from data and MC result in 2-20\% uncertainties across the various search bins. Other systematic uncertainties arise from the geometrical and kinematic acceptance for the muons (3\%), and the $\tau$-jet response function (1-15\%). An uncertainty of 1-8\% is assigned to account for possible differences between data and MC simulation for the acceptance of the $m_{\mathrm{T}}$ selection. 

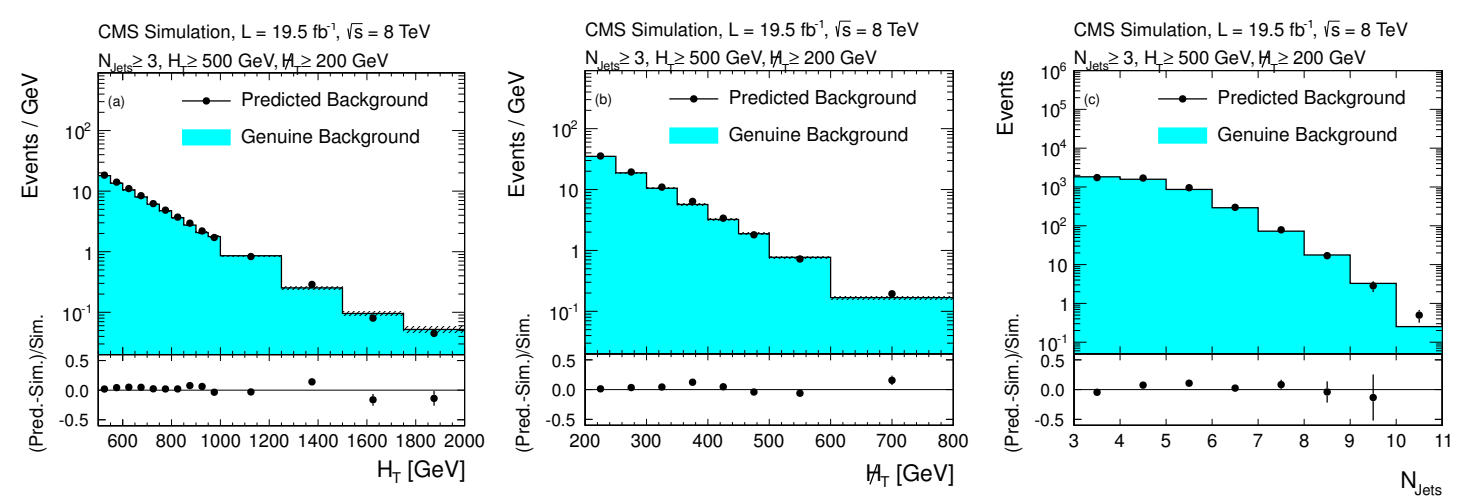

Figure 3. Predicted (a) $H_{\mathrm{T}}$, (b) $H_{\mathrm{T}}$, and (c) $N_{\text {Jets }}$ distributions found from applying the $\tau_{\mathrm{h}}$ background evaluation method to simulated $t \bar{t}$ and $\mathrm{W}+$ jets events (solid points) in comparison to the genuine $t \overline{\mathrm{t}}$ and $\mathrm{W}+$ jets background from simulation (shaded curve). Only statistical uncertainties are shown.

\subsection{Estimation of the QCD multijet background}

The background from QCD multijet events is evaluated with the "rebalance-and-smear" method [9, 10], using data samples recorded with $H_{\mathrm{T}}$ thresholds ranging from 350 to $650 \mathrm{GeV}$. The events, recorded with a trigger prescaled by a factor $k$, are sampled $k$ times to create seed events as described below.

In the rebalance step, the momenta of the jets with $p_{\mathrm{T}}>10 \mathrm{GeV} / c$ in each event are adjusted within the jet- $p_{\mathrm{T}}$-resolution values, using a kinematic fit, such that the events are balanced in the transverse plane. Considering only jets with $p_{\mathrm{T}}$ above a certain threshold introduces an additional imbalance in the event, which results in larger $p_{\mathrm{T}}$ for the rebalanced jets than the expected true value. This effect is compensated by scaling the rebalanced jets by a $p_{\mathrm{T}}$-dependent factor derived by comparing rebalanced and generator-level jets in the simulation. The scaling factors derived using either PYTHIA or MADGRAPH, and with different average pileup interactions, are found to be similar. The jets in the rebalanced events are then smeared using jet $p_{\mathrm{T}}$ response functions, which are obtained from MC simulation as a function of $p_{\mathrm{T}}$ and $\eta$, and adjusted to match those determined from dijet and $\gamma+$ jets data [27]. The QCD multijet background is predicted by applying selection criteria on the kinematic quantities calculated from the smeared jets. The procedure is repeated one hundred times to evaluate the average prediction and its statistical uncertainty in each search region.

The method is validated using simulated QCD multijet events. Comparisons of the $H_{\mathrm{T}}$, $H_{\mathrm{T}}$, and $N_{\text {Jets }}$ distributions from the MC simulation to those predicted by the rebalanceand-smear method on the same simulated events are shown in figure 4. A systematic uncertainty of $11-86 \%$ is assigned based on the statistical precision attributed to the validation procedure, which is performed both in the search regions and in QCD-enriched data control regions defined either by $100<H_{\mathrm{T}}<200 \mathrm{GeV}$ or by inverting the $\left|\Delta \phi\left(\vec{p}_{\mathrm{T}}^{\text {Jet1,2,3 }}, \vec{H}_{\mathrm{T}}\right)\right|$ selection. Due to the limited number of events in individual search bins, this uncertainty is evaluated for each jet multiplicity bin for $H_{\mathrm{T}}$ smaller or larger than $1000 \mathrm{GeV}$, inclu- 

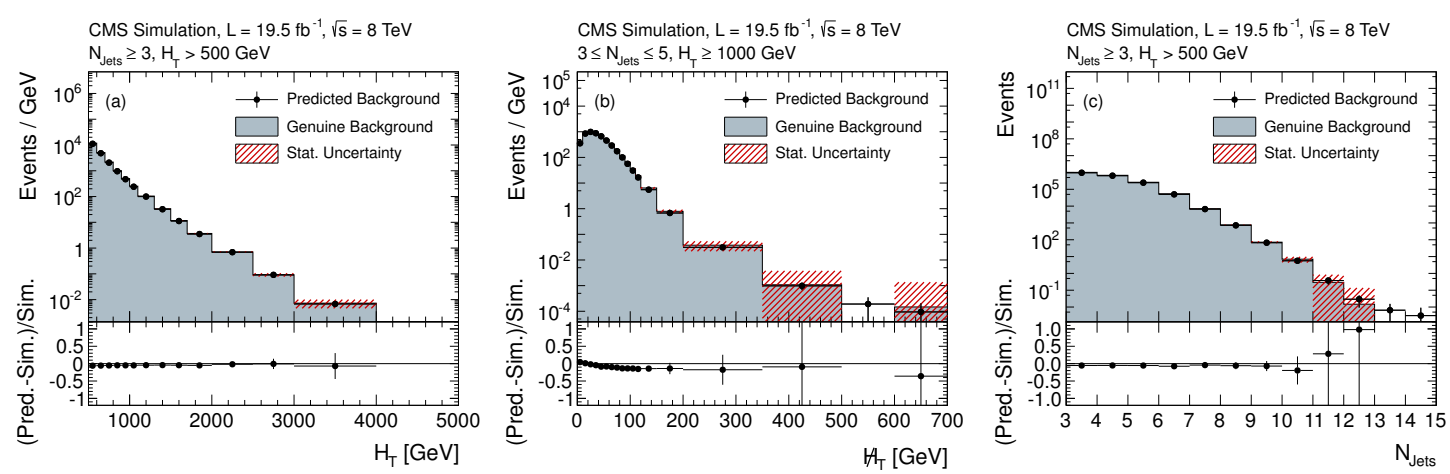

Figure 4. Predicted (a) $H_{\mathrm{T}}$, (b) $H_{\mathrm{T}}$, and (c) $N_{\text {Jets }}$ distributions found from applying the "rebalance-and-smear" method to simulated QCD multijet events (solid points) in comparison with the genuine QCD multijet background from simulation (shaded curve). The distributions are shown for events that satisfy the baseline selection, except that the $\mathscr{H}_{\mathrm{T}}$ selection is not applied, and in addition $H_{\mathrm{T}}>1000 \mathrm{GeV}$ is required for the events used in the $H_{\mathrm{T}}$ distribution. The statistical uncertainties are indicated by the hatched band for the expectation and by error bars for the prediction.

sive over $H_{\mathrm{T}}$. The uncertainty due to differences in the core and tails of the jet response functions between data and simulation results in uncertainties of $10-30 \%$ and $20-35 \%$, respectively. An uncertainty of $3 \%, 8 \%$, and $35 \%$ is assigned for search regions with $N_{\text {Jets }}$ $=3-5,6-7$, and $\geq 8$, respectively, to account for the effect of pileup. The predicted QCD multijet background contributions to the search bins along with associated uncertainties are given in table 1.

\section{$5 \quad$ Results and interpretation}

The predicted background event yields and the number of observed events are summarized in table 1 and figure 5 for the 36 search regions. The data are consistent with the expected background contributions from SM processes. A slight excess of events is observed in the search bin with $N_{\text {Jets }}=6-7, H_{\mathrm{T}}=500-800 \mathrm{GeV}$, and $H_{\mathrm{T}}>450 \mathrm{GeV}$, which is insignificant when the probability to observe a statistical fluctuation as large or larger in any of the search regions is considered.

The results are interpreted in the context of simplified models $[21,22]$ of pair production of squarks $(\widetilde{\mathrm{q}})$ or gluinos $(\widetilde{\mathrm{g}})$. These particles decay directly, or via intermediate new particles, to quarks and an LSP, where the LSP is denoted as $\widetilde{\chi}_{1}^{0}$ in the following. The signal events are generated at LO using MADGRAPH5, with up to two additional partons. The cross sections are determined at NLO and include the resummation of soft gluon emission at the accuracy of next-to-leading-log (NLL) calculations [50-55]. Both for the generation of signal events and the calculation of $\widetilde{\mathrm{q}}(\widetilde{\mathrm{g}})$ production cross section, the contribution of $\widetilde{\mathrm{g}}(\widetilde{\mathrm{q}})$ production is effectively removed by assuming the gluino (squark) mass to be very large.

Several decay modes of gluinos are considered here, $\widetilde{\mathrm{g}} \rightarrow \mathrm{q} \overline{\mathrm{q}}+\widetilde{\chi}_{1}^{0}, \widetilde{\mathrm{g}} \rightarrow \mathrm{t} \overline{\mathrm{t}}+\widetilde{\chi}_{1}^{0}$, and $\widetilde{\mathrm{g}} \rightarrow \mathrm{q} \overline{\mathrm{q}}+\widetilde{\chi}_{1}^{ \pm} / \widetilde{\chi}_{2}^{0}$ where $\widetilde{\chi}_{1}^{ \pm} \rightarrow \mathrm{W}+\widetilde{\chi}_{1}^{0}$ and $\widetilde{\chi}_{2}^{0} \rightarrow \mathrm{Z}+\widetilde{\chi}_{1}^{0}$. The branching fraction for 


\begin{tabular}{|c|c|c|c|c|c|c|c|c|}
\hline \multicolumn{3}{|c|}{ Selection } & \multirow[t]{2}{*}{$\mathrm{Z} \rightarrow \nu \bar{\nu}$} & \multirow{2}{*}{$\begin{array}{c}\mathrm{t} \overline{\mathrm{t}} / \mathrm{W} \\
\rightarrow \mathrm{e}, \mu+\mathrm{X}\end{array}$} & \multirow{2}{*}{$\begin{array}{c}\mathrm{t} \overline{\mathrm{t}} / \mathrm{W} \\
\rightarrow \tau_{\mathrm{h}}+\mathrm{X}\end{array}$} & \multirow[t]{2}{*}{ QCD } & \multirow{2}{*}{$\begin{array}{c}\text { Total } \\
\text { background }\end{array}$} & \multirow[t]{2}{*}{ Data } \\
\hline$N_{\text {Jets }}$ & $H_{\mathrm{T}}[\mathrm{GeV}]$ & $\not H_{\mathrm{T}}[\mathrm{GeV}]$ & & & & & & \\
\hline $3-5$ & $500-800$ & $200-300$ & $1820 \pm 390$ & $2210 \pm 450$ & $1750 \pm 210$ & $310 \pm 220$ & $6090 \pm 670$ & 6159 \\
\hline $3-5$ & 500-800 & $300-450$ & $990 \pm 220$ & $660 \pm 130$ & $590 \pm 70$ & $40 \pm 20$ & $2280 \pm 270$ & 2305 \\
\hline $3-5$ & $500-800$ & $450-600$ & $273 \pm 63$ & $77 \pm 17$ & $66.3 \pm 9.5$ & $1.3_{-1.3}^{+1.5}$ & $418 \pm 66$ & 454 \\
\hline $3-5$ & $500-800$ & $>600$ & $42 \pm 10$ & $9.5 \pm 4.0$ & $5.7 \pm 1.3$ & $0.1_{-0.1}^{+0.3}$ & $57.4 \pm 11.2$ & 62 \\
\hline $3-5$ & $800-1000$ & $200-300$ & $216 \pm 46$ & $278 \pm 62$ & $192 \pm 33$ & $92 \pm 66$ & $777 \pm 107$ & 808 \\
\hline $3-5$ & $800-1000$ & $300-450$ & $124 \pm 26$ & $113 \pm 27$ & $84 \pm 12$ & $9.9 \pm 7.4$ & $330 \pm 40$ & 305 \\
\hline $3-5$ & 800-1000 & $450-600$ & $47 \pm 11$ & $36.1 \pm 9.9$ & $24.1 \pm 3.6$ & $0.8_{-0.8}^{+1.3}$ & $108 \pm 15$ & 124 \\
\hline $3-5$ & 800-1000 & $>600$ & $35.3 \pm 8.8$ & $9.0 \pm 3.7$ & $10.3 \pm 2.0$ & $0.1_{-0.1}^{+0.4}$ & $54.8 \pm 9.7$ & 52 \\
\hline $3-5$ & $1000-1250$ & $200-300$ & $76 \pm 17$ & $104 \pm 26$ & $66.5 \pm 9.9$ & $59 \pm 25$ & $305 \pm 41$ & 335 \\
\hline $3-5$ & $1000-1250$ & $300-450$ & $39.3 \pm 8.9$ & $52 \pm 14$ & $41 \pm 11$ & $5.1 \pm 2.7$ & 137 & 129 \\
\hline $3-5$ & $1000-1250$ & $450-600$ & $18.1 \pm 4.7$ & $6.9 \pm 3.2$ & $6.8 \pm 2.0$ & $0.5_{-0.5}^{+0.7}$ & $32.3=$ & 34 \\
\hline $3-5$ & $1000-1250$ & $>600$ & $17.8 \pm 4.8$ & $2.4 \pm 1.8$ & $2.5 \pm 0.8$ & $0.1_{-0.1}^{+0.3}$ & $22.8 \pm 5.2$ & 32 \\
\hline $3-5$ & $1250-1500$ & $200-300$ & $25.3 \pm 6.0$ & $31.0 \pm 9.5$ & $21.3 \pm 4.1$ & $31 \pm 13$ & $109 \pm 18$ & 98 \\
\hline $3-5$ & & $300-450$ & $16.7 \pm$ & $10.1 \pm 4.4$ & $13.7 \pm 7.1$ & $2.3 \pm$ & 42.8 & 38 \\
\hline $3-5$ & $1250-1500$ & $>450$ & $12.3 \pm 3.5$ & $2.3 \pm 1.7$ & $2.7 \pm 1.2$ & $0.2_{-0.2}^{+0.5}$ & $17.6 \pm 4.1$ & 23 \\
\hline $3-5$ & $>1500$ & $200-300$ & $10.5 \pm 2.9$ & $16.7 \pm 6.2$ & $23.5 \pm 5.6$ & $35 \pm 14$ & & 94 \\
\hline $3-5$ & $>1500$ & $>300$ & $10.9 \pm 3.1$ & $9.7 \pm 4.3$ & $6.6 \pm 1.4$ & $2.4 \pm 2.0$ & $29.7 \pm$ & 39 \\
\hline 6-7 & $500-800$ & $200-300$ & $22.7 \pm 6.4$ & $133 \pm 59$ & $117 \pm 25$ & $18.2 \pm 9.2$ & $290 \pm 65$ & 266 \\
\hline $6-7$ & $500-\varepsilon$ & & .2 & & $18.0 \pm 5.1$ & $1.9 \pm$ & & 62 \\
\hline $6-7$ & 500-800 & $>450$ & $0.7 \pm 0.6$ & $0.0_{-0.0}^{+3.2}$ & $0.1_{-0.1}^{+0.5}$ & $0.0_{-0.0}^{+0.1}$ & $0.8_{-0.6}^{+3.3}$ & 9 \\
\hline $6-7$ & & & $9.1 \pm 3$ & $56 \pm 25$ & $46 \pm 11$ & $13.1 \pm 6.6$ & 124 & 111 \\
\hline $6-7$ & $800-1000$ & $300-450$ & $4.2 \pm 1.7$ & $10.4 \pm 5.5$ & $12.0 \pm 3.6$ & $1.9 \pm 1.4$ & $28.6 \pm 6.9$ & 35 \\
\hline $6-7$ & 800-1000 & $>450$ & $1.8 \pm 1.0$ & $2.9 \pm 2.5$ & $1.2 \pm 0.8$ & $0.1_{-0.1}^{+0.4}$ & $6.0 \pm 2.8$ & 4 \\
\hline $6-7$ & $1000-1250$ & $200-300$ & $4.4 \pm 1.7$ & $24 \pm 12$ & $29.5 \pm 7.8$ & $11.9 \pm 6.0$ & $70 \pm 16$ & 67 \\
\hline 6-7 & $1000-1250$ & & & & & $1.5 \pm 1.5$ & $21.6 \pm$ & 20 \\
\hline $6-7$ & $1000-1250$ & $>450$ & $1.4 \pm 0.8$ & $0.0_{-0.0}^{+3.6}$ & $0.6_{-0.6}^{+0.8}$ & $0.1_{-0.1}^{+0.4}$ & $2.2_{-1.1}^{+3.8}$ & 4 \\
\hline 6-7 & $1250-1500$ & $200-300$ & $3.3 \pm 1.4$ & $11.5 \pm 6.5$ & $6.4 \pm 2.7$ & $6.8 \pm 3.9$ & $28.0 \pm 8.2$ & 24 \\
\hline 6-7 & $1250-1500$ & $300-450$ & $1.4 \pm 0.8$ & $3.5 \pm 2.6$ & $3.5 \pm 1.9$ & $0.9_{-0.9}^{+1.3}$ & $9.4 \pm 3.6$ & 5 \\
\hline $6-7$ & $1250-1500$ & $>450$ & $0.4 \pm 0.4$ & $0.0_{-0.0}^{+2.5}$ & $0.1_{-0.1}^{+0.5}$ & $0.1_{-0.1}^{+0.3}$ & $0.5_{-0.4}^{+2.6}$ & 2 \\
\hline $6-7$ & $>1500$ & $200-300$ & $1.3 \pm 0.8$ & $10.0 \pm 6.9$ & $2.0 \pm 1.2$ & $7.8 \pm 4.0$ & $21.1 \pm 8.1$ & 18 \\
\hline $6-7$ & $>1500$ & $>300$ & $1.1 \pm 0.7$ & $3.2 \pm 2.8$ & $2.8 \pm 1.9$ & $0.8_{-0.8}^{+1.1}$ & $7.9 \pm 3.6$ & 3 \\
\hline$\geq 8$ & $500-800$ & $>200$ & $0.0_{-0.0}^{+0.8}$ & $1.9 \pm 1.5$ & $2.8 \pm 1.4$ & $0.1_{-0.1}^{+0.4}$ & $4.8_{-2.1}^{+2.3}$ & 8 \\
\hline$\geq 8$ & $800-1000$ & $>200$ & $0.6 \pm 0.6$ & & $2.3 \pm 1.2$ & & & 9 \\
\hline$\geq 8$ & $1000-1250$ & $>200$ & $0.6 \pm 0.5$ & $1.4_{-1.4}^{+1.5}$ & $2.9 \pm 1.3$ & $0.7_{-0.7}^{+1.0}$ & $5.6_{-2.1}^{+2.3}$ & 8 \\
\hline$\geq 8$ & $1250-1500$ & $>200$ & & & $1.4 \pm 0.9$ & & & 5 \\
\hline$\geq 8$ & $>1500$ & $>200$ & $0.0_{-0.0}^{+0.7}$ & $0.0_{-0.0}^{+4.2}$ & $2.4 \pm 1.4$ & $0.9_{-0.9}^{+1.3}$ & $3.3_{-1.7}^{+4.7}$ & 2 \\
\hline
\end{tabular}

Table 1. Predicted event yields for the different background components in the search regions defined by $H_{\mathrm{T}}, H_{\mathrm{T}}$ and $N_{\text {Jets }}$. The uncertainties of the different background sources are added in quadrature to obtain the total uncertainties. 


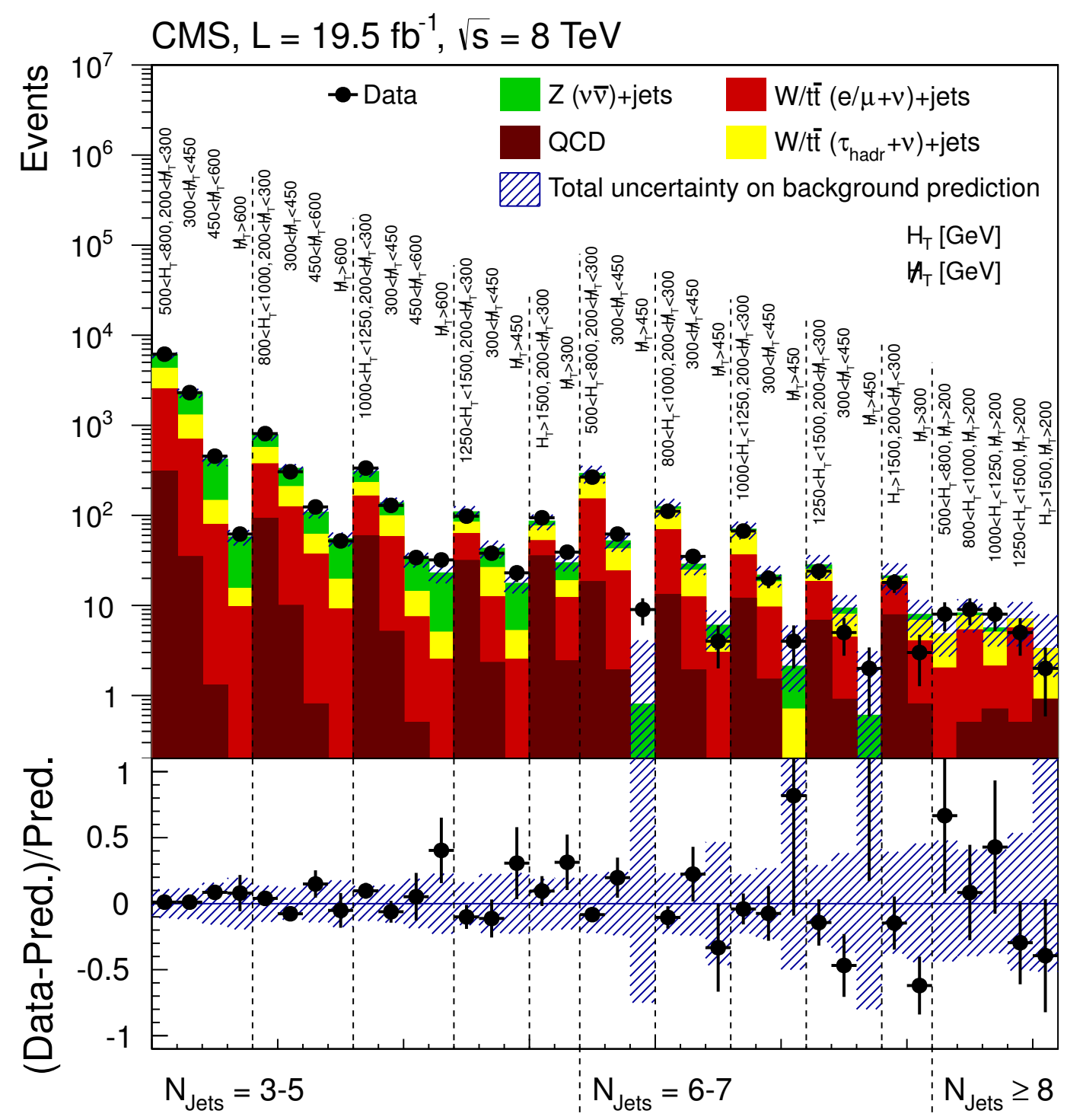

Figure 5. Summary of the observed number of events in each of the 36 search regions in comparison to the corresponding background prediction. The hatched region shows the total uncertainty of the background prediction.

the different decay modes is assumed, in turn, to be $100 \%$, except for the $\widetilde{\mathrm{g}} \rightarrow \mathrm{q} \overline{\mathrm{q}}+\widetilde{\chi}$ process, where the decay proceeds via $\widetilde{\chi}_{1}^{+}, \widetilde{\chi}_{1}^{-}$and $\widetilde{\chi}_{2}^{0}$ particles with equal probability. Squark production is studied in the decay mode $\widetilde{\mathrm{q}} \rightarrow \mathrm{q}+\widetilde{\chi}_{1}^{0}$. The models are studied in the parameter space of the mass of the LSP versus the mass of the gluino or squark. The $H_{\mathrm{T}}$ distributions observed for the three intervals of jet multiplicity are shown in figure 6 in comparison to the SM background prediction. The $\not_{\mathrm{T}}$ distributions expected from gluino or squark pair production are overlaid for $m_{\widetilde{\mathrm{g}}}=1.1 \mathrm{TeV}$ and $m_{\widetilde{\chi}_{1}^{0}}=125 \mathrm{GeV}$, and for $m_{\widetilde{\mathrm{q}}}$ $=700 \mathrm{GeV}$ and $m_{\widetilde{\chi}_{1}^{0}}=100 \mathrm{GeV}$, in various decay modes.

The $95 \%$ confidence level (CL) upper limits on the signal production cross section are set using the LHC-style $\mathrm{CL}_{\mathrm{s}}$ criterion [56-58]. The signal acceptance and efficiencies, and 

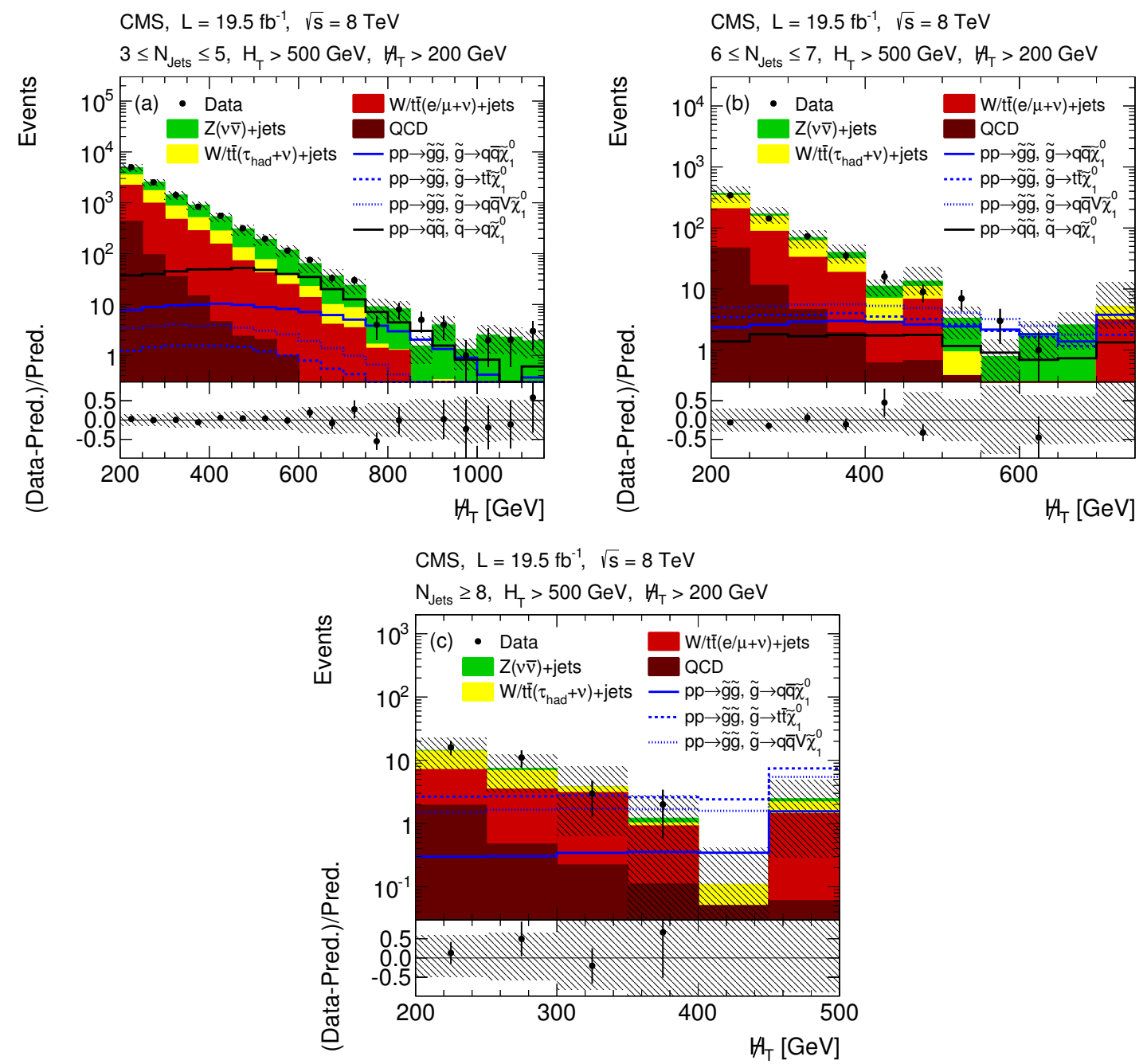

Figure 6. Observed $H_{\mathrm{T}}$ distributions compared to the predicted backgrounds for search regions with $H_{\mathrm{T}}>500 \mathrm{GeV}$ and jet multiplicity intervals of (a) $3-5$, (b) $6-7$, and (c) $\geq 8$. The background distributions are stacked. The last bin contains the overflow. The hatched region indicates the uncertainties of the background predictions. The ratio of data to the background is shown in the lower plots. The $H_{\mathrm{T}}$ distributions expected from events with $\widetilde{\mathrm{g}}$ and $\widetilde{\mathrm{q}}$ pair production, with either $m_{\widetilde{\mathrm{g}}}=1.1 \mathrm{TeV}$ and $m_{\widetilde{\chi}_{1}^{0}}=125 \mathrm{GeV}$ or $m_{\widetilde{\mathrm{q}}}=700 \mathrm{GeV}$ and $m_{\widetilde{\chi}_{1}^{0}}=100 \mathrm{GeV}$, are overlaid.

corresponding uncertainties for the 36 exclusive search regions, along with the background estimates discussed above, are combined into a likelihood that is used to construct the test statistic based on the profile likelihood ratio. The uncertainties of the signal acceptance and efficiency due to several sources are taken into account when cross section upper limits are determined. The uncertainties due to the luminosity determination (2.6\%) [59], trigger inefficiency $(2 \%)$, and event cleaning procedure (3\%) [28] are the same for all signal models and search regions. The uncertainty from the measurement of the jet energy scale and jet energy resolution [27] leads to uncertainties of $2-8 \%$ and $1-2 \%$ in signal acceptance. The variation of PDFs [47] results in 1-8\% uncertainty from the signal acceptance. The rate 

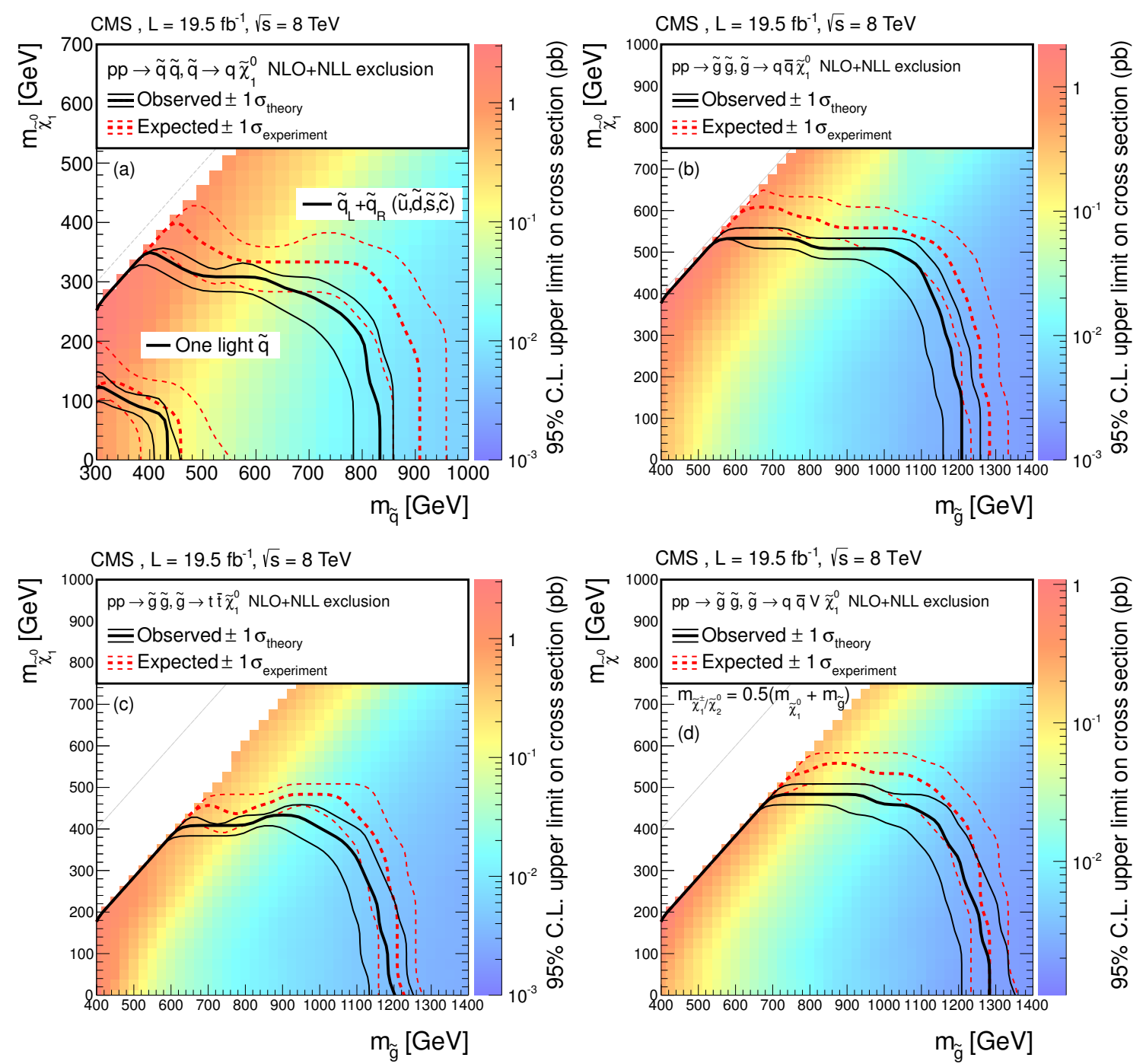

Figure 7. The observed and expected 95\% CL upper limits on the (a) $\widetilde{\mathrm{q}} \widetilde{\mathrm{q}}$ and (b-d) $\widetilde{\mathrm{g}} \widetilde{\mathrm{g}}$ production cross sections in either the $\left(m_{\widetilde{\mathrm{q}}}, m_{\widetilde{\chi}_{1}^{0}}\right)$ or the $\left(m_{\widetilde{\mathrm{g}}}, m_{\widetilde{\chi}_{1}^{0}}\right)$ plane obtained with the simplified models. For the $\widetilde{\mathrm{q}} \widetilde{\mathrm{q}}$ production the upper set of curves corresponds to the scenario when the first two generations of squarks are degenerate and light, while the lower set corresponds to only one light accessible squark.

of initial-state radiation in the signal event simulation is corrected to correspond to that measured in data [60], leading to a corresponding uncertainty of $22 \%$ for model points with small differences between the masses of the gluino or squark and the $\widetilde{\chi}_{1}^{0}$. For larger mass differences, this uncertainty is typically less than a few percent.

The observed and expected 95\% CL upper limits on the signal cross section are shown for the production of a $\widetilde{\mathrm{q}} \widetilde{\mathrm{q}}$ pair with $\widetilde{\mathrm{q}} \rightarrow \mathrm{q}+\widetilde{\chi}_{1}^{0}$ in figure $7(\mathrm{a})$, a $\widetilde{g} \widetilde{g}$ pair with $\widetilde{\mathrm{g}} \rightarrow$ $\mathrm{q} \overline{\mathrm{q}}+\widetilde{\chi}_{1}^{0}$ in figure $7(\mathrm{~b})$, a $\widetilde{\mathrm{g}} \widetilde{\mathrm{g}}$ pair with $\widetilde{\mathrm{g}} \rightarrow \mathrm{t} \overline{\mathrm{t}}+\widetilde{\chi}_{1}^{0}$ in figure $7(\mathrm{c})$, and a $\widetilde{\mathrm{g} g}$ pair with $\widetilde{\mathrm{g}} \rightarrow \mathrm{q} \overline{\mathrm{q}}+\mathrm{W} / \mathrm{Z}+\widetilde{\chi}_{1}^{0}$ in figure $7(\mathrm{~d})$, in the $\left(m_{\widetilde{\mathrm{q}}}, m_{\widetilde{\chi}_{1}^{0}}\right)$ and $\left(m_{\widetilde{\mathrm{g}}}, m_{\widetilde{\chi}_{1}^{0}}\right)$ planes. The contours show the exclusion regions for the signal production cross sections obtained using the 
NLO+NLL calculations. The exclusion contours are also presented when the signal cross section is varied by changing the renormalization and factorization scales by a factor of two and using the PDF uncertainty based on the CTEQ6.6 [61] and MSTW2008 [62] PDF sets. Conservatively, by comparing the observed limit to the theoretical cross section minus its one-standard-deviation uncertainty, for the cases where the gluino decays as $\widetilde{\mathrm{g}} \rightarrow \mathrm{q} \overline{\mathrm{q}}+\widetilde{\chi}_{1}^{0}$, $\widetilde{\mathrm{g}} \rightarrow \mathrm{t} \overline{\mathrm{t}}+\widetilde{\chi}_{1}^{0}$, and $\widetilde{\mathrm{g}} \rightarrow \mathrm{q} \overline{\mathrm{q}}+\mathrm{W} / \mathrm{Z}+\widetilde{\chi}_{1}^{0}$, gluino masses up to $1.16,1.13$, and $1.21 \mathrm{TeV}$ are excluded, respectively, for $m_{\widetilde{\chi}_{1}^{0}}<100 \mathrm{GeV}$. For direct $\widetilde{\mathrm{q}} \widetilde{\mathrm{q}}$ production of the first two generations of squarks $\left(\widetilde{\mathrm{u}}_{L / R}, \widetilde{\mathrm{d}}_{L / R}, \widetilde{\mathrm{c}}_{L / R}, \widetilde{\mathrm{s}}_{L / R}\right)$, values of $m_{\widetilde{\mathrm{q}}}$ below $780 \mathrm{GeV}$ are excluded for $m_{\widetilde{\chi}_{1}^{0}}<200 \mathrm{GeV}$. If only one of these squarks is light, then $m_{\widetilde{\mathrm{q}}}$ values below $400 \mathrm{GeV}$ are excluded for $m_{\widetilde{\chi}_{1}^{0}}<80 \mathrm{GeV}$. The expected search sensitivity is improved with respect to our similar analysis [10] based on the $7 \mathrm{TeV}$ data set by up to about $200 \mathrm{GeV}$ in the values of $m_{\widetilde{\mathrm{g}}}, m_{\widetilde{\mathrm{q}}}$ and $m_{\widetilde{\chi}_{1}^{0}}$.

\section{Summary}

An inclusive search for supersymmetry has been performed in multijet events with $N_{\text {Jets }}=$ $3-5,6-7$, and $\geq 8$, and large missing transverse momentum. The data sample corresponds to an integrated luminosity of $19.5 \mathrm{fb}^{-1}$ collected in $8 \mathrm{TeV}$ pp collisions during the year 2012 with the CMS detector at the LHC. The analysis extends the supersymmetric parameter space explored by searches in the all-hadronic final state. The observed numbers of events are found to be consistent with the expected standard model background, which is evaluated from the data. The results are presented in the context of simplified models, where final states are described by the pair production of new particles decaying to one, two, or more jets and a weakly interacting stable neutral particle, e.g. the lightest supersymmetric particle (LSP). Squark masses below $780 \mathrm{GeV}$ and gluino masses of up to 1.1-1.2 TeV are excluded at 95\% CL within the studied models for LSP masses below $100 \mathrm{GeV}$.

\section{Acknowledgments}

We congratulate our colleagues in the CERN accelerator departments for the excellent performance of the LHC and thank the technical and administrative staffs at CERN and at other CMS institutes for their contributions to the success of the CMS effort. In addition, we gratefully acknowledge the computing centres and personnel of the Worldwide LHC Computing Grid for delivering so effectively the computing infrastructure essential to our analyses. Finally, we acknowledge the enduring support for the construction and operation of the LHC and the CMS detector provided by the following funding agencies: BMWF and FWF (Austria); FNRS and FWO (Belgium); CNPq, CAPES, FAPERJ, and FAPESP (Brazil); MES (Bulgaria); CERN; CAS, MoST, and NSFC (China); COLCIENCIAS (Colombia); MSES and CSF (Croatia); RPF (Cyprus); MoER, SF0690030s09 and ERDF (Estonia); Academy of Finland, MEC, and HIP (Finland); CEA and CNRS/IN2P3 (France); BMBF, DFG, and HGF (Germany); GSRT (Greece); OTKA and NIH (Hungary); DAE and DST (India); IPM (Iran); SFI (Ireland); INFN (Italy); NRF and WCU (Republic of Korea); LAS (Lithuania); MOE and UM (Malaysia); CINVESTAV, CONACYT, SEP, 
and UASLP-FAI (Mexico); MBIE (New Zealand); PAEC (Pakistan); MSHE and NSC (Poland); FCT (Portugal); JINR (Dubna); MON, RosAtom, RAS and RFBR (Russia); MESTD (Serbia); SEIDI and CPAN (Spain); Swiss Funding Agencies (Switzerland); NSC (Taipei); ThEPCenter, IPST, STAR and NSTDA (Thailand); TUBITAK and TAEK (Turkey); NASU (Ukraine); STFC (United Kingdom); DOE and NSF (U.S.A.).

Individuals have received support from the Marie-Curie programme and the European Research Council and EPLANET (European Union); the Leventis Foundation; the A. P. Sloan Foundation; the Alexander von Humboldt Foundation; the Belgian Federal Science Policy Office; the Fonds pour la Formation à la Recherche dans l'Industrie et dans l'Agriculture (FRIA-Belgium); the Agentschap voor Innovatie door Wetenschap en Technologie (IWT-Belgium); the Ministry of Education, Youth and Sports (MEYS) of Czech Republic; the Council of Science and Industrial Research, India; the Compagnia di San Paolo (Torino); the HOMING PLUS programme of Foundation for Polish Science, cofinanced by EU, Regional Development Fund; and the Thalis and Aristeia programmes cofinanced by EU-ESF and the Greek NSRF.

Open Access. This article is distributed under the terms of the Creative Commons Attribution License (CC-BY 4.0), which permits any use, distribution and reproduction in any medium, provided the original author(s) and source are credited.

\section{References}

[1] ATLAS collaboration, Observation of a new particle in the search for the Standard Model Higgs boson with the ATLAS detector at the LHC, Phys. Lett. B 716 (2012) 1 [arXiv: 1207.7214] [INSPIRE].

[2] CMS collaboration, Observation of a new boson at a mass of $125 \mathrm{GeV}$ with the CMS experiment at the LHC, Phys. Lett. B 716 (2012) 30 [arXiv:1207.7235] [INSPIRE].

[3] CMS collaboration, Observation of a new boson with mass near $125 \mathrm{GeV}$ in pp collisions at $\sqrt{s}=7$ and $8 \mathrm{TeV}$, JHEP 06 (2013) 081 [arXiv:1303.4571] [INSPIRE].

[4] G.R. Farrar and P. Fayet, Phenomenology of the Production, Decay and Detection of New Hadronic States Associated with Supersymmetry, Phys. Lett. B 76 (1978) 575 [INSPIRE].

[5] CMS collaboration, The CMS experiment at the CERN LHC, 2008 JINST 3 S08004 [INSPIRE].

[6] J. Wess and B. Zumino, Supergauge Transformations in Four-Dimensions, Nucl. Phys. B 70 (1974) 39 [INSPIRE].

[7] H.-C. Cheng and I. Low, TeV symmetry and the little hierarchy problem, JHEP 09 (2003) 051 [hep-ph/0308199] [INSPIRE].

[8] T. Appelquist, H.-C. Cheng and B.A. Dobrescu, Bounds on universal extra dimensions, Phys. Rev. D 64 (2001) 035002 [hep-ph/0012100] [INSPIRE].

[9] CMS collaboration, Search for New Physics with Jets and Missing Transverse Momentum in pp collisions at $\sqrt{s}=7 \mathrm{TeV}$, JHEP 08 (2011) 155 [arXiv:1106.4503] [INSPIRE]. 
[10] CMS collaboration, Search for new physics in the multijet and missing transverse momentum final state in proton-proton collisions at $\sqrt{s}=7$ TeV, Phys. Rev. Lett. 109 (2012) 171803 [arXiv: 1207.1898] [INSPIRE].

[11] CMS collaboration, Search for supersymmetry in hadronic final states using MT2 in pp collisions at $\sqrt{s}=7 \mathrm{TeV}$, JHEP 10 (2012) 018 [arXiv:1207.1798] [INSPIRE].

[12] CMS collaboration, Search for supersymmetry in hadronic final states with missing transverse energy using the variables $\alpha_{T}$ and b-quark multiplicity in pp collisions at $\sqrt{s}=8$ TeV, Eur. Phys. J. C 73 (2013) 2568 [arXiv:1303.2985] [InSPIRE].

[13] CMS collaboration, Search for gluino mediated bottom-and top-squark production in multijet final states in pp collisions at 8 TeV, Phys. Lett. B 725 (2013) 243 [arXiv:1305.2390] [INSPIRE].

[14] ATLAS collaboration, Search for squarks and gluinos with the ATLAS detector in final states with jets and missing transverse momentum using $4.7 \mathrm{fb}^{-1}$ of $\sqrt{\mathrm{s}}=7 \mathrm{TeV}$ proton-proton collision data, Phys. Rev. D 87 (2013) 012008 [arXiv:1208.0949] [InSPIRE].

[15] ATLAS collaboration, Hunt for new phenomena using large jet multiplicities and missing transverse momentum with ATLAS in $4.7 \mathrm{fb}^{-1}$ of $\sqrt{s}=7$ TeV proton-proton collisions, JHEP 07 (2012) 167 [arXiv:1206.1760] [INSPIRE].

[16] ATLAS collaboration, Search for new phenomena in final states with large jet multiplicities and missing transverse momentum at $\sqrt{s}=8 \mathrm{TeV}$ proton-proton collisions using the ATLAS experiment, JHEP 10 (2013) 130 [arXiv: 1308.1841] [INSPIRE].

[17] ATLAS collaboration, Search for direct third-generation squark pair production in final states with missing transverse momentum and two b-jets in $\sqrt{s}=8 \mathrm{TeV}$ pp collisions with the ATLAS detector, JHEP 10 (2013) 189 [arXiv:1308.2631] [INSPIRE].

[18] A.H. Chamseddine, R.L. Arnowitt and P. Nath, Locally Supersymmetric Grand Unification, Phys. Rev. Lett. 49 (1982) 970 [InSPIRE].

[19] R.L. Arnowitt and P. Nath, SUSY mass spectrum in SU(5) supergravity grand unification, Phys. Rev. Lett. 69 (1992) 725 [INSPIRE].

[20] G.L. Kane, C.F. Kolda, L. Roszkowski and J.D. Wells, Study of constrained minimal supersymmetry, Phys. Rev. D 49 (1994) 6173 [hep-ph/9312272] [InSPIRE].

[21] J. Alwall, P. Schuster and N. Toro, Simplified Models for a First Characterization of New Physics at the LHC, Phys. Rev. D 79 (2009) 075020 [arXiv:0810.3921] [InSPIRE].

[22] CMS collaboration, Interpretation of Searches for Supersymmetry with simplified Models, Phys. Rev. D 88 (2013) 052017 [arXiv: 1301.2175] [InSPIRE].

[23] CMS collaboration, Particle-Flow Event Reconstruction in CMS and Performance for Jets, Taus and MET, CMS Physics Analysis Summary, CMS-PAS-PFT-09-001 (2009).

[24] M. Cacciari, G.P. Salam and G. Soyez, The anti- $k_{T}$ jet clustering algorithm, JHEP 04 (2008) 063 [arXiv:0802.1189] [INSPIRE].

[25] M. Cacciari and G.P. Salam, Pileup subtraction using jet areas, Phys. Lett. B 659 (2008) 119 [arXiv:0707.1378] [INSPIRE].

[26] M. Cacciari, G.P. Salam and G. Soyez, The catchment area of jets, JHEP 04 (2008) 005 [arXiv:0802.1188] [INSPIRE]. 
[27] CMS collaboration, Determination of Jet Energy Calibration and Transverse Momentum Resolution in CMS, 2011 JINST 6 P11002 [arXiv:1107.4277] [INSPIRE].

[28] CMS collaboration, Missing transverse energy performance of the CMS detector, 2011 JINST 6 P09001 [arXiv:1106.5048] [INSPIRE].

[29] CMS collaboration, MET performance in 8 TeV data, CMS Physics Analysis Summary, CMS-PAS-JME-12-002 (2013).

[30] CMS collaboration, Electron reconstruction and identification at $\sqrt{s}=7 \mathrm{TeV}$, CMS Physics Analysis Summary, CMS-PAS-EGM-10-004 (2010).

[31] CMS collaboration, Performance of CMS muon reconstruction in pp collision events at $\sqrt{s}=7 \mathrm{TeV}, 2012$ JINST 7 P10002 [arXiv:1206.4071] [INSPIRE].

[32] J. Alwall et al., MadGraph/MadEvent v4: The New Web Generation, JHEP 09 (2007) 028 [arXiv:0706.2334] [INSPIRE].

[33] T. Sjöstrand, S. Mrenna and P.Z. Skands, PYTHIA 6.4 Physics and Manual, JHEP 05 (2006) 026 [hep-ph/0603175] [inSPIRE].

[34] N. Kidonakis, Next-to-next-to-leading soft-gluon corrections for the top quark cross section and transverse momentum distribution, Phys. Rev. D 82 (2010) 114030 [arXiv:1009.4935] [INSPIRE].

[35] K. Melnikov and F. Petriello, Electroweak gauge boson production at hadron colliders through O $\left(\alpha_{s}^{2}\right)$, Phys. Rev. D 74 (2006) 114017 [hep-ph/0609070] [INSPIRE].

[36] S. Agostinelli et al., GEANT4 - a simulation toolkit, Nucl. Instrum. Meth. 506 (2003) 250.

[37] J. Pumplin et al., New generation of parton distributions with uncertainties from global QCD analysis, JHEP 07 (2002) 012 [hep-ph/0201195] [INSPIRE].

[38] CMS collaboration, The fast simulation of the CMS detector at LHC, J. Phys. Conf. Ser. 331 (2011) 032049 [INSPIRE].

[39] R. Field, Early LHC Underlying Event Data - Findings and Surprises, arXiv:1010.3558 [INSPIRE].

[40] Z. Bern et al., Driving Missing Data at Next-to-Leading Order, Phys. Rev. D 84 (2011) 114002 [arXiv: 1106.1423] [inSPIRE].

[41] J.H. Kuhn, A. Kulesza, S. Pozzorini and M. Schulze, Electroweak corrections to hadronic photon production at large transverse momenta, JHEP 03 (2006) 059 [hep-ph/0508253] [INSPIRE].

[42] S. Ask, M.A. Parker, T. Sandoval, M.E. Shea and W.J. Stirling, Using $\gamma+$ jets Production to Calibrate the Standard Model $Z(\rightarrow \nu \bar{\nu})+$ jets Background to New Physics Processes at the LHC, JHEP 10 (2011) 058 [arXiv:1107.2803] [inSPIRE].

[43] Z. Bern et al., Missing Energy and Jets for Supersymmetry Searches, Phys. Rev. D 87 (2013) 034026 [arXiv: 1206.6064] [INSPIRE].

[44] CMS collaboration, Photon reconstruction and identification at $\sqrt{s}=7 \mathrm{TeV}$, CMS Physics Analysis Summary, CMS-PAS-EGM-10-005 (2010).

[45] CMS collaboration, Energy calibration and resolution of the CMS electromagnetic calorimeter in pp collisions at $\sqrt{s}=7 \mathrm{TeV}, 2013$ JINST 8 P09009 [arXiv:1306.2016] [INSPIRE]. 
[46] CMS collaboration, Measurement of the Inclusive $W$ and $Z$ Production Cross sections in pp Collisions at $\sqrt{s}=7 \mathrm{TeV}$, JHEP 10 (2011) 132 [arXiv:1107.4789] [INSPIRE].

[47] M. Botje et al., The PDF 4 LHC Working Group Interim Recommendations, arXiv: 1101.0538 [INSPIRE].

[48] Particle Data Group collaboration, J. Beringer et al., Review of Particle Physics (RPP), Phys. Rev. D 86 (2012) 010001 [InSPIRE].

[49] B. Efron, The Jackknife, the Bootstrap and other Resampling Plans, SIAM, Philadelphia, U.S.A., CBMS-NSF Regional Conference Series in Applied Mathematics 38 (1982).

[50] W. Beenakker, R. Hopker, M. Spira and P.M. Zerwas, Squark and gluino production at hadron colliders, Nucl. Phys. B 492 (1997) 51 [hep-ph/9610490] [INSPIRE].

[51] A. Kulesza and L. Motyka, Threshold resummation for squark-antisquark and gluino-pair production at the LHC, Phys. Rev. Lett. 102 (2009) 111802 [arXiv:0807.2405] [INSPIRE].

[52] A. Kulesza and L. Motyka, Soft gluon resummation for the production of gluino-gluino and squark-antisquark pairs at the LHC, Phys. Rev. D 80 (2009) 095004 [arXiv: 0905.4749] [INSPIRE].

[53] W. Beenakker et al., Soft-gluon resummation for squark and gluino hadroproduction, JHEP 12 (2009) 041 [arXiv: 0909.4418] [INSPIRE].

[54] W. Beenakker et al., Squark and Gluino Hadroproduction, Int. J. Mod. Phys. A 26 (2011) 2637 [arXiv: 1105.1110] [INSPIRE].

[55] M. Krämer et al., Supersymmetry production cross sections in pp collisions at $\sqrt{s}=7 \mathrm{TeV}$, arXiv:1206.2892 [INSPIRE].

[56] A.L. Read, Presentation of search results: The CL(s) technique, J. Phys. G 28 (2002) 2693 [INSPIRE].

[57] T. Junk, Confidence level computation for combining searches with small statistics, Nucl. Instrum. Meth. A 434 (1999) 435 [hep-ex/9902006] [INSPIRE].

[58] ATLAS, CMS collaborations, Procedure for the LHC Higgs boson search combination in summer 2011, ATL-PHYS-PUB-2011-011 [CMS-NOTE-2011-005] (2011).

[59] CMS collaboration, CMS Luminosity Based on Pixel Cluster Counting - Summer 2013 Update, CMS Physics Analysis Summary, CMS-PAS-LUM-13-001 (2013).

[60] CMS collaboration, Search for top-squark pair production in the single-lepton final state in pp collisions at $\sqrt{s}=8 \mathrm{TeV}$, Eur. Phys. J. C 73 (2013) 2677 [arXiv:1308.1586] [inSPIRE].

[61] P.M. Nadolsky et al., Implications of CTEQ global analysis for collider observables, Phys. Rev. D 78 (2008) 013004 [arXiv: 0802.0007] [INSPIRE].

[62] A.D. Martin, W.J. Stirling, R.S. Thorne and G. Watt, Parton distributions for the LHC, Eur. Phys. J. C 63 (2009) 189 [arXiv:0901.0002] [inSPIRE]. 


\section{The CMS collaboration}

\section{Yerevan Physics Institute, Yerevan, Armenia}

S. Chatrchyan, V. Khachatryan, A.M. Sirunyan, A. Tumasyan

Institut für Hochenergiephysik der OeAW, Wien, Austria

W. Adam, T. Bergauer, M. Dragicevic, J. Erö, C. Fabjan ${ }^{1}$, M. Friedl, R. Frühwirth ${ }^{1}$, V.M. Ghete, C. Hartl, N. Hörmann, J. Hrubec, M. Jeitler ${ }^{1}$, W. Kiesenhofer, V. Knünz, M. Krammer ${ }^{1}$, I. Krätschmer, D. Liko, I. Mikulec, D. Rabady² , B. Rahbaran, H. Rohringer, R. Schöfbeck, J. Strauss, A. Taurok, W. Treberer-Treberspurg, W. Waltenberger, C.-E. Wulz ${ }^{1}$

\section{National Centre for Particle and High Energy Physics, Minsk, Belarus}

V. Mossolov, N. Shumeiko, J. Suarez Gonzalez

\section{Universiteit Antwerpen, Antwerpen, Belgium}

S. Alderweireldt, M. Bansal, S. Bansal, T. Cornelis, E.A. De Wolf, X. Janssen, A. Knutsson, S. Luyckx, L. Mucibello, S. Ochesanu, B. Roland, R. Rougny, H. Van Haevermaet, P. Van Mechelen, N. Van Remortel, A. Van Spilbeeck

\section{Vrije Universiteit Brussel, Brussel, Belgium}

F. Blekman, S. Blyweert, J. D'Hondt, N. Heracleous, A. Kalogeropoulos, J. Keaveney, T.J. Kim, S. Lowette, M. Maes, A. Olbrechts, D. Strom, S. Tavernier, W. Van Doninck, P. Van Mulders, G.P. Van Onsem, I. Villella

\section{Université Libre de Bruxelles, Bruxelles, Belgium}

C. Caillol, B. Clerbaux, G. De Lentdecker, L. Favart, A.P.R. Gay, A. Léonard, P.E. Marage, A. Mohammadi, L. Perniè, T. Reis, T. Seva, L. Thomas, C. Vander Velde, P. Vanlaer, J. Wang

\section{Ghent University, Ghent, Belgium}

V. Adler, K. Beernaert, L. Benucci, A. Cimmino, S. Costantini, S. Dildick, G. Garcia, B. Klein, J. Lellouch, J. Mccartin, A.A. Ocampo Rios, D. Ryckbosch, S. Salva Diblen, M. Sigamani, N. Strobbe, F. Thyssen, M. Tytgat, S. Walsh, E. Yazgan, N. Zaganidis

Université Catholique de Louvain, Louvain-la-Neuve, Belgium

S. Basegmez, C. Beluffi ${ }^{3}$, G. Bruno, R. Castello, A. Caudron, L. Ceard, G.G. Da Silveira, C. Delaere, T. du Pree, D. Favart, L. Forthomme, A. Giammanco ${ }^{4}$, J. Hollar, P. Jez, M. Komm, V. Lemaitre, J. Liao, O. Militaru, C. Nuttens, D. Pagano, A. Pin, K. Piotrzkowski, A. Popov ${ }^{5}$, L. Quertenmont, M. Selvaggi, M. Vidal Marono, J.M. Vizan Garcia

Université de Mons, Mons, Belgium

N. Beliy, T. Caebergs, E. Daubie, G.H. Hammad

Centro Brasileiro de Pesquisas Fisicas, Rio de Janeiro, Brazil

G.A. Alves, M. Correa Martins Junior, T. Martins, M.E. Pol, M.H.G. Souza 
Universidade do Estado do Rio de Janeiro, Rio de Janeiro, Brazil

W.L. Aldá Júnior, W. Carvalho, J. Chinellato ${ }^{6}$, A. Custódio, E.M. Da Costa, D. De Jesus Damiao, C. De Oliveira Martins, S. Fonseca De Souza, H. Malbouisson, M. Malek, D. Matos Figueiredo, L. Mundim, H. Nogima, W.L. Prado Da Silva, J. Santaolalla, A. Santoro, A. Sznajder, E.J. Tonelli Manganote ${ }^{6}$, A. Vilela Pereira

Universidade Estadual Paulista ${ }^{a}$, Universidade Federal do $\mathbf{A B C}^{b}$, São Paulo, Brazil

C.A. Bernardes ${ }^{b}$, F.A. Dias ${ }^{a, 7}$, T.R. Fernandez Perez Tomei ${ }^{a}$, E.M. Gregores ${ }^{b}$, P.G. Mercadante ${ }^{b}$, S.F. Novaes ${ }^{a}$, Sandra S. Padula ${ }^{a}$

Institute for Nuclear Research and Nuclear Energy, Sofia, Bulgaria

V. Genchev², P. Iaydjiev², A. Marinov, S. Piperov, M. Rodozov, G. Sultanov, M. Vutova University of Sofia, Sofia, Bulgaria

A. Dimitrov, I. Glushkov, R. Hadjiiska, V. Kozhuharov, L. Litov, B. Pavlov, P. Petkov

Institute of High Energy Physics, Beijing, China

J.G. Bian, G.M. Chen, H.S. Chen, M. Chen, R. Du, C.H. Jiang, D. Liang, S. Liang, X. Meng, R. Plestina ${ }^{8}$, J. Tao, X. Wang, Z. Wang

State Key Laboratory of Nuclear Physics and Technology, Peking University, Beijing, China

C. Asawatangtrakuldee, Y. Ban, Y. Guo, Q. Li, W. Li, S. Liu, Y. Mao, S.J. Qian, D. Wang,

L. Zhang, W. Zou

Universidad de Los Andes, Bogota, Colombia

C. Avila, C.A. Carrillo Montoya, L.F. Chaparro Sierra, C. Florez, J.P. Gomez,

B. Gomez Moreno, J.C. Sanabria

Technical University of Split, Split, Croatia

N. Godinovic, D. Lelas, D. Polic, I. Puljak

University of Split, Split, Croatia

Z. Antunovic, M. Kovac

Institute Rudjer Boskovic, Zagreb, Croatia

V. Brigljevic, K. Kadija, J. Luetic, D. Mekterovic, S. Morovic, L. Tikvica

University of Cyprus, Nicosia, Cyprus

A. Attikis, G. Mavromanolakis, J. Mousa, C. Nicolaou, F. Ptochos, P.A. Razis

Charles University, Prague, Czech Republic

M. Finger, M. Finger Jr.

Academy of Scientific Research and Technology of the Arab Republic of Egypt, Egyptian Network of High Energy Physics, Cairo, Egypt

A.A. Abdelalim ${ }^{9}$, Y. Assran ${ }^{10}$, S. Elgammal ${ }^{11}$, A. Ellithi Kamel ${ }^{12}$, M.A. Mahmoud ${ }^{13}$, A. Radi ${ }^{11,14}$ 
National Institute of Chemical Physics and Biophysics, Tallinn, Estonia

M. Kadastik, M. Müntel, M. Murumaa, M. Raidal, L. Rebane, A. Tiko

Department of Physics, University of Helsinki, Helsinki, Finland

P. Eerola, G. Fedi, M. Voutilainen

Helsinki Institute of Physics, Helsinki, Finland

J. Härkönen, V. Karimäki, R. Kinnunen, M.J. Kortelainen, T. Lampén, K. Lassila-Perini,

S. Lehti, T. Lindén, P. Luukka, T. Mäenpää, T. Peltola, E. Tuominen, J. Tuominiemi,

E. Tuovinen, L. Wendland

Lappeenranta University of Technology, Lappeenranta, Finland

T. Tuuva

DSM/IRFU, CEA/Saclay, Gif-sur-Yvette, France

M. Besancon, F. Couderc, M. Dejardin, D. Denegri, B. Fabbro, J.L. Faure, F. Ferri,

S. Ganjour, A. Givernaud, P. Gras, G. Hamel de Monchenault, P. Jarry, E. Locci, J. Malcles, A. Nayak, J. Rander, A. Rosowsky, M. Titov

Laboratoire Leprince-Ringuet, Ecole Polytechnique, IN2P3-CNRS, Palaiseau, France

S. Baffioni, F. Beaudette, P. Busson, C. Charlot, N. Daci, T. Dahms, M. Dalchenko, L. Dobrzynski, A. Florent, R. Granier de Cassagnac, P. Miné, C. Mironov, I.N. Naranjo, M. Nguyen, C. Ochando, P. Paganini, D. Sabes, R. Salerno, J.b. Sauvan, Y. Sirois, C. Veelken, Y. Yilmaz, A. Zabi

Institut Pluridisciplinaire Hubert Curien, Université de Strasbourg, Université de Haute Alsace Mulhouse, CNRS/IN2P3, Strasbourg, France

J.-L. Agram ${ }^{15}$, J. Andrea, D. Bloch, J.-M. Brom, E.C. Chabert, C. Collard, E. Conte ${ }^{15}$, F. Drouhin ${ }^{15}$, J.-C. Fontaine ${ }^{15}$, D. Gelé, U. Goerlach, C. Goetzmann, P. Juillot, A.-C. Le Bihan, P. Van Hove

Centre de Calcul de l'Institut National de Physique Nucleaire et de Physique des Particules, CNRS/IN2P3, Villeurbanne, France

S. Gadrat

Université de Lyon, Université Claude Bernard Lyon 1, CNRS-IN2P3, Institut de Physique Nucléaire de Lyon, Villeurbanne, France

S. Beauceron, N. Beaupere, G. Boudoul, S. Brochet, J. Chasserat, R. Chierici, D. Contardo ${ }^{2}$, P. Depasse, H. El Mamouni, J. Fan, J. Fay, S. Gascon, M. Gouzevitch, B. Ille, T. Kurca, M. Lethuillier, L. Mirabito, S. Perries, J.D. Ruiz Alvarez, L. Sgandurra, V. Sordini, M. Vander Donckt, P. Verdier, S. Viret, H. Xiao

Institute of High Energy Physics and Informatization, Tbilisi State University, Tbilisi, Georgia

Z. Tsamalaidze ${ }^{16}$ 


\section{RWTH Aachen University, I. Physikalisches Institut, Aachen, Germany}

C. Autermann, S. Beranek, M. Bontenackels, B. Calpas, M. Edelhoff, L. Feld, O. Hindrichs, K. Klein, A. Ostapchuk, A. Perieanu, F. Raupach, J. Sammet, S. Schael, D. Sprenger, H. Weber, B. Wittmer, V. Zhukov ${ }^{5}$

\section{RWTH Aachen University, III. Physikalisches Institut A, Aachen, Germany}

M. Ata, J. Caudron, E. Dietz-Laursonn, D. Duchardt, M. Erdmann, R. Fischer, A. Güth, T. Hebbeker, C. Heidemann, K. Hoepfner, D. Klingebiel, S. Knutzen, P. Kreuzer, M. Merschmeyer, A. Meyer, M. Olschewski, K. Padeken, P. Papacz, H. Reithler, S.A. Schmitz, L. Sonnenschein, D. Teyssier, S. Thüer, M. Weber

\section{RWTH Aachen University, III. Physikalisches Institut B, Aachen, Germany}

V. Cherepanov, Y. Erdogan, G. Flügge, H. Geenen, M. Geisler, W. Haj Ahmad, F. Hoehle, B. Kargoll, T. Kress, Y. Kuessel, J. Lingemann ${ }^{2}$, A. Nowack, I.M. Nugent, L. Perchalla, O. Pooth, A. Stahl

\section{Deutsches Elektronen-Synchrotron, Hamburg, Germany}

I. Asin, N. Bartosik, J. Behr, W. Behrenhoff, U. Behrens, A.J. Bell, M. Bergholz ${ }^{17}$, A. Bethani, K. Borras, A. Burgmeier, A. Cakir, L. Calligaris, A. Campbell, S. Choudhury, F. Costanza, C. Diez Pardos, S. Dooling, T. Dorland, G. Eckerlin, D. Eckstein, T. Eichhorn, G. Flucke, A. Geiser, A. Grebenyuk, P. Gunnellini, S. Habib, J. Hauk, G. Hellwig, M. Hempel, D. Horton, H. Jung, M. Kasemann, P. Katsas, J. Kieseler, C. Kleinwort, M. Krämer, D. Krücker, W. Lange, J. Leonard, K. Lipka, W. Lohmann ${ }^{17}$, B. Lutz, R. Mankel, I. Marfin, I.-A. Melzer-Pellmann, A.B. Meyer, J. Mnich, A. Mussgiller, S. Naumann-Emme, O. Novgorodova, F. Nowak, H. Perrey, A. Petrukhin, D. Pitzl, R. Placakyte, A. Raspereza, P.M. Ribeiro Cipriano, C. Riedl, E. Ron, M.Ö. Sahin, J. Salfeld-Nebgen, P. Saxena, R. Schmidt ${ }^{17}$, T. Schoerner-Sadenius, M. Schröder, M. Stein, A.D.R. Vargas Trevino, R. Walsh, C. Wissing

\section{University of Hamburg, Hamburg, Germany}

M. Aldaya Martin, V. Blobel, A.R. Draeger, H. Enderle, J. Erfle, E. Garutti, K. Goebel, M. Görner, M. Gosselink, J. Haller, R.S. Höing, H. Kirschenmann, R. Klanner, R. Kogler, J. Lange, T. Lapsien, T. Lenz, I. Marchesini, J. Ott, T. Peiffer, N. Pietsch, D. Rathjens, C. Sander, H. Schettler, P. Schleper, E. Schlieckau, A. Schmidt, M. Seidel, J. Sibille ${ }^{18}$, V. Sola, H. Stadie, G. Steinbrück, D. Troendle, E. Usai, L. Vanelderen

\section{Institut für Experimentelle Kernphysik, Karlsruhe, Germany}

C. Barth, C. Baus, J. Berger, C. Böser, E. Butz, T. Chwalek, W. De Boer, A. Descroix, A. Dierlamm, M. Feindt, M. Guthoff ${ }^{2}$, F. Hartmann ${ }^{2}$, T. Hauth ${ }^{2}$, H. Held, K.H. Hoffmann, U. Husemann, I. Katkov ${ }^{5}$, A. Kornmayer ${ }^{2}$, E. Kuznetsova, P. Lobelle Pardo, D. Martschei, M.U. Mozer, Th. Müller, M. Niegel, A. Nürnberg, O. Oberst, G. Quast, K. Rabbertz, F. Ratnikov, S. Röcker, F.-P. Schilling, G. Schott, H.J. Simonis, F.M. Stober, R. Ulrich, J. Wagner-Kuhr, S. Wayand, T. Weiler, R. Wolf, M. Zeise 
Institute of Nuclear and Particle Physics (INPP), NCSR Demokritos, Aghia Paraskevi, Greece

G. Anagnostou, G. Daskalakis, T. Geralis, S. Kesisoglou, A. Kyriakis, D. Loukas, A. Markou, C. Markou, E. Ntomari, A. Psallidas, I. Topsis-giotis

University of Athens, Athens, Greece

L. Gouskos, A. Panagiotou, N. Saoulidou, E. Stiliaris

University of Ioánnina, Ioánnina, Greece

X. Aslanoglou, I. Evangelou, G. Flouris, C. Foudas, J. Jones, P. Kokkas, N. Manthos, I. Papadopoulos, E. Paradas

Wigner Research Centre for Physics, Budapest, Hungary

G. Bencze, C. Hajdu, P. Hidas, D. Horvath ${ }^{19}$, F. Sikler, V. Veszpremi, G. Vesztergombi ${ }^{20}$, A.J. Zsigmond

Institute of Nuclear Research ATOMKI, Debrecen, Hungary

N. Beni, S. Czellar, J. Molnar, J. Palinkas, Z. Szillasi

University of Debrecen, Debrecen, Hungary

J. Karancsi, P. Raics, Z.L. Trocsanyi, B. Ujvari

National Institute of Science Education and Research, Bhubaneswar, India

S.K. Swain

Panjab University, Chandigarh, India

S.B. Beri, V. Bhatnagar, N. Dhingra, R. Gupta, M. Kaur, M.Z. Mehta, M. Mittal, N. Nishu, A. Sharma, J.B. Singh

University of Delhi, Delhi, India

Ashok Kumar, Arun Kumar, S. Ahuja, A. Bhardwaj, B.C. Choudhary, A. Kumar, S. Malhotra, M. Naimuddin, K. Ranjan, V. Sharma, R.K. Shivpuri

Saha Institute of Nuclear Physics, Kolkata, India

S. Banerjee, S. Bhattacharya, K. Chatterjee, S. Dutta, B. Gomber, Sa. Jain, Sh. Jain, R. Khurana, A. Modak, S. Mukherjee, D. Roy, S. Sarkar, M. Sharan, A.P. Singh

Bhabha Atomic Research Centre, Mumbai, India

A. Abdulsalam, D. Dutta, S. Kailas, V. Kumar, A.K. Mohanty², L.M. Pant, P. Shukla, A. Topkar

Tata Institute of Fundamental Research - EHEP, Mumbai, India

T. Aziz, R.M. Chatterjee, S. Ganguly, S. Ghosh, M. Guchait ${ }^{21}$, A. Gurtu ${ }^{22}$, G. Kole, S. Kumar, M. Maity ${ }^{23}$, G. Majumder, K. Mazumdar, G.B. Mohanty, B. Parida, K. Sudhakar, N. Wickramage ${ }^{24}$

Tata Institute of Fundamental Research - HECR, Mumbai, India

S. Banerjee, S. Dugad 
Institute for Research in Fundamental Sciences (IPM), Tehran, Iran

H. Arfaei, H. Bakhshiansohi, H. Behnamian, S.M. Etesami ${ }^{25}$, A. Fahim ${ }^{26}$, A. Jafari, M. Khakzad, M. Mohammadi Najafabadi, M. Naseri, S. Paktinat Mehdiabadi, B. Safarzadeh ${ }^{27}$, M. Zeinali

\section{University College Dublin, Dublin, Ireland}

M. Grunewald

INFN Sezione di Bari ${ }^{a}$, Università di Bari ${ }^{b}$, Politecnico di Bari ${ }^{c}$, Bari, Italy

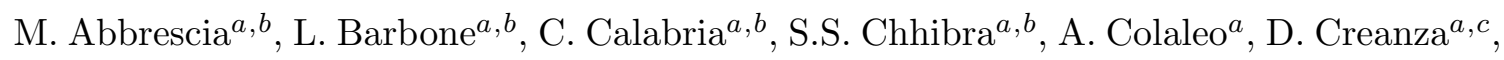
N. De Filippis ${ }^{a, c}$, M. De Palma ${ }^{a, b}$, L. Fiore ${ }^{a}$, G. Iaselli ${ }^{a, c}$, G. Maggi ${ }^{a, c}$, M. Maggi $^{a}$, B. Marangelli ${ }^{a, b}$, S. My ${ }^{a, c}$, S. Nuzzo ${ }^{a, b}$, N. Pacifico ${ }^{a}$, A. Pompili ${ }^{a}, b$, G. Pugliese ${ }^{a, c}$, R. Radogna ${ }^{a, b}$, G. Selvaggi ${ }^{a, b}$, L. Silvestris ${ }^{a}$, G. Singh ${ }^{a}, b$, R. Venditti ${ }^{a}, b$, P. Verwilligen ${ }^{a}$, G. Zito ${ }^{a}$

\section{INFN Sezione di Bologna ${ }^{a}$, Università di Bologna ${ }^{b}$, Bologna, Italy}

G. Abbiendi $^{a}$, A.C. Benvenuti ${ }^{a}$, D. Bonacorsi ${ }^{a}, b$ S. Braibant-Giacomelli ${ }^{a, b}$, L. Brigliadori ${ }^{a, b}$, R. Campanini ${ }^{a, b}$, P. Capiluppi ${ }^{a, b}$, A. Castro ${ }^{a, b}$, F.R. Cavallo ${ }^{a}$,

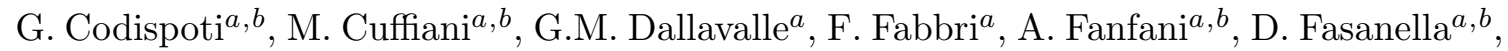
P. Giacomelli ${ }^{a}$, C. Grandi ${ }^{a}$, L. Guiducci ${ }^{a}, b$, S. Marcellini ${ }^{a}$, G. Masetti ${ }^{a}$, M. Meneghelli $^{a, b}$,

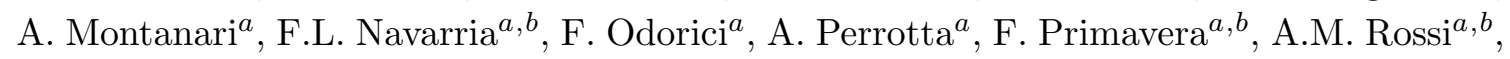
T. Rovelli ${ }^{a, b}$, G.P. Siroli ${ }^{a, b}$, N. Tosi ${ }^{a, b}$, R. Travaglini ${ }^{a, b}$

INFN Sezione di Catania ${ }^{a}$, Università di Catania ${ }^{b}, \operatorname{CSFNSM}^{c}$, Catania, Italy

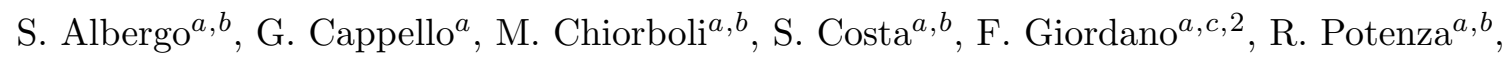
A. Tricomi ${ }^{a, b}$, C. Tuve ${ }^{a, b}$

INFN Sezione di Firenze ${ }^{a}$, Università di Firenze ${ }^{b}$, Firenze, Italy

G. Barbagli ${ }^{a}$, V. Ciulli ${ }^{a, b}$, C. Civinini ${ }^{a}$, R. D’Alessandro ${ }^{a, b}$, E. Focardi ${ }^{a, b}$, E. Gallo ${ }^{a}$, S. Gonzi ${ }^{a, b}$, V. Gori ${ }^{a, b}$, P. Lenzi ${ }^{a, b}$, M. Meschini ${ }^{a}$, S. Paoletti ${ }^{a}$, G. Sguazzoni ${ }^{a}$, A. Tropiano ${ }^{a, b}$

INFN Laboratori Nazionali di Frascati, Frascati, Italy

L. Benussi, S. Bianco, F. Fabbri, D. Piccolo

INFN Sezione di Genova ${ }^{a}$, Università di Genova ${ }^{b}$, Genova, Italy

P. Fabbricatore ${ }^{a}$, R. Ferretti ${ }^{a, b}$, F. Ferro ${ }^{a}$, M. Lo Vetere ${ }^{a, b}$, R. Musenich ${ }^{a}$, E. Robutti ${ }^{a}$, S. Tosi ${ }^{a, b}$

INFN Sezione di Milano-Bicocca ${ }^{a}$, Università di Milano-Bicocca ${ }^{b}$, Milano, Italy

A. Benaglia ${ }^{a}$, M.E. Dinardo ${ }^{a, b}$, S. Fiorendi ${ }^{a, b, 2}$, S. Gennai ${ }^{a}$, R. Gerosa, A. Ghezzi ${ }^{a, b}$, P. Govoni ${ }^{a, b}$, M.T. Lucchini ${ }^{a, b, 2}$, S. Malvezzi ${ }^{a}$, R.A. Manzoni ${ }^{a, b, 2}$, A. Martellia ${ }^{a, b}, 2$, B. Marzocchi, D. Menasce ${ }^{a}$, L. Moroni ${ }^{a}$, M. Paganoni ${ }^{a, b}$, D. Pedrini ${ }^{a}$, S. Ragazzi ${ }^{a}, b$, N. Redaelli ${ }^{a}$, T. Tabarelli de Fatis ${ }^{a, b}$ 
INFN Sezione di Napoli ${ }^{a}$, Università di Napoli 'Federico II ${ }^{b}$, Università della Basilicata (Potenza) ${ }^{c}$, Università G. Marconi (Roma) ${ }^{d}$, Napoli, Italy

S. Buontempo ${ }^{a}$, N. Cavallo ${ }^{a, c}$, S. Di Guida ${ }^{a, d}$, F. Fabozzi ${ }^{a, c}$, A.O.M. Iorio $^{a, b}$, L. Lista $^{a}$, S. Meola ${ }^{a, d, 2}$, M. Merola ${ }^{a}$, P. Paolucci ${ }^{a, 2}$

INFN Sezione di Padova ${ }^{a}$, Università di Padova ${ }^{b}$, Università di Trento (Trento) ${ }^{c}$, Padova, Italy

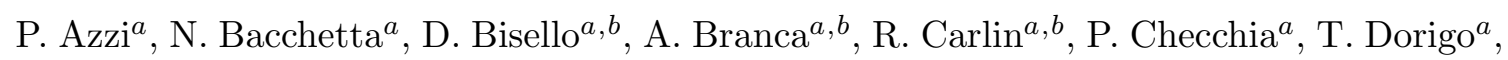
U. Dosselli ${ }^{a}$, M. Galanti ${ }^{a, b, 2}$, F. Gasparini ${ }^{a, b}$, U. Gasparini ${ }^{a}, b$, P. Giubilato ${ }^{a, b}$, F. Gonella ${ }^{a}$, A. Gozzelino ${ }^{a}$, K. Kanishchev ${ }^{a, c}$, S. Lacaprara ${ }^{a}$, I. Lazzizzera ${ }^{a, c}$, M. Margoni ${ }^{a, b}$, A.T. Meneguzzo ${ }^{a, b}$, F. Montecassiano ${ }^{a}$, J. Pazzini ${ }^{a, b}$, N. Pozzobon ${ }^{a, b}$, P. Ronchese $^{a, b}$,

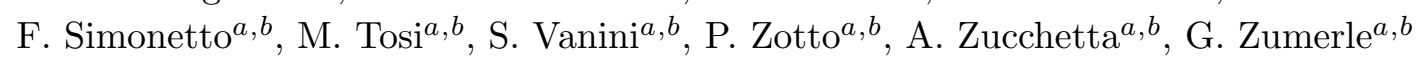

INFN Sezione di Pavia ${ }^{a}$, Università di Pavia ${ }^{b}$, Pavia, Italy

M. Gabusi ${ }^{a}, b$, S.P. Ratti ${ }^{a, b}$, C. Riccardi ${ }^{a, b}$, P. Vitulo ${ }^{a, b}$

INFN Sezione di Perugia ${ }^{a}$, Università di Perugia ${ }^{b}$, Perugia, Italy

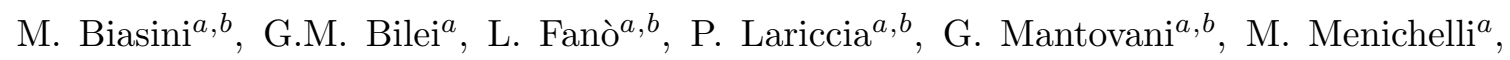
F. Romeo ${ }^{a, b}$, A. Saha ${ }^{a}$, A. Santocchia ${ }^{a, b}$, A. Spiezia ${ }^{a, b}$

INFN Sezione di Pisa ${ }^{a}$, Università di Pisa ${ }^{b}$, Scuola Normale Superiore di Pisa ${ }^{c}$, Pisa, Italy

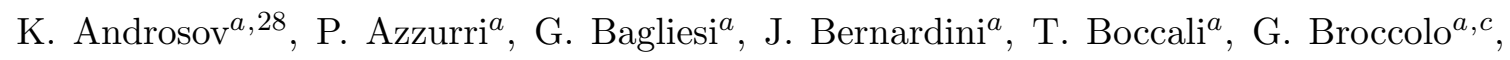
R. Castaldi ${ }^{a}$, M.A. Ciocci ${ }^{a, 28}$, R. Dell'Orso ${ }^{a}$, F. Fiori ${ }^{a, c}$, L. Foà ${ }^{a, c}$, A. Giassi ${ }^{a}$,

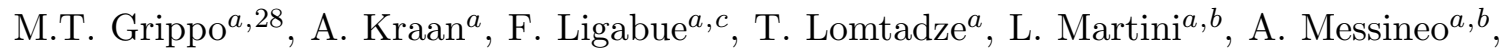
C.S. Moon ${ }^{a, 29}$, F. Palla ${ }^{a}$, A. Rizzi ${ }^{a, b}$, A. Savoy-Navarro ${ }^{a, 30}$, A.T. $\operatorname{Serban}^{a}$, P. Spagnolo ${ }^{a}$,

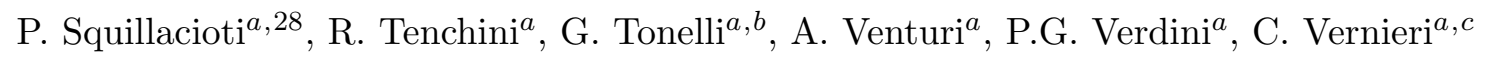

INFN Sezione di Roma ${ }^{a}$, Università di Roma ${ }^{b}$, Roma, Italy

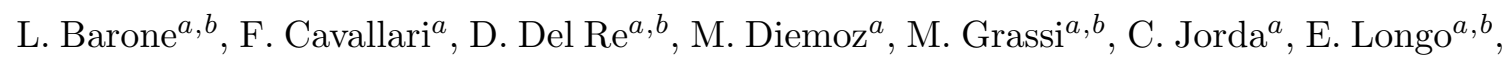
F. Margaroli ${ }^{a, b}$, P. Meridiani ${ }^{a}$, F. Micheli ${ }^{a, b}$, S. Nourbakhsh ${ }^{a, b}$, G. Organtini ${ }^{a, b}$, R. Paramatti ${ }^{a}$, S. Rahatlou ${ }^{a, b}$, C. Rovelli ${ }^{a}$, L. Soffi ${ }^{a, b}$, P. Traczyk ${ }^{a, b}$

INFN Sezione di Torino ${ }^{a}$, Università di Torino ${ }^{b}$, Università del Piemonte Orientale (Novara) ${ }^{c}$, Torino, Italy

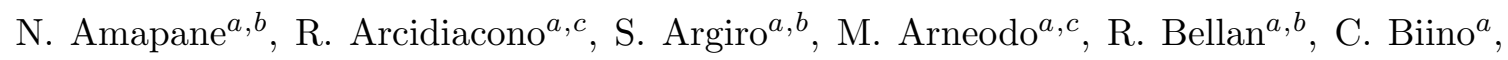
N. Cartiglia ${ }^{a}$, S. Casasso ${ }^{a, b}$, M. Costa ${ }^{a, b}$, A. Degano ${ }^{a, b}$, N. Demaria ${ }^{a}$, C. Mariotti $^{a}$,

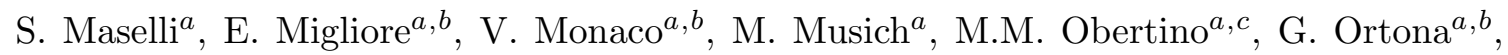
L. Pacher ${ }^{a, b}$, N. Pastrone ${ }^{a}$, M. Pelliccioni ${ }^{a, 2}$, A. Potenza ${ }^{a, b}$, A. Romero ${ }^{a, b}$, M. Ruspa ${ }^{a, c}$, R. Sacchi ${ }^{a, b}$, A. Solano ${ }^{a, b}$, A. Staiano $^{a}$, U. Tamponi $^{a}$

INFN Sezione di Trieste ${ }^{a}$, Università di Trieste ${ }^{b}$, Trieste, Italy

S. Belforte ${ }^{a}$, V. Candelise ${ }^{a, b}$, M. Casarsa ${ }^{a}$, F. Cossutti ${ }^{a}$, G. Della Ricca ${ }^{a, b}$, B. Gobbo ${ }^{a}$,

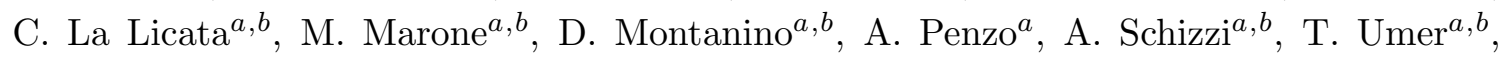
A. Zanetti ${ }^{a}$ 
Kangwon National University, Chunchon, Korea

S. Chang, T.Y. Kim, S.K. Nam

Kyungpook National University, Daegu, Korea

D.H. Kim, G.N. Kim, J.E. Kim, M.S. Kim, D.J. Kong, S. Lee, Y.D. Oh, H. Park, D.C. Son

Chonnam National University, Institute for Universe and Elementary Particles, Kwangju, Korea

J.Y. Kim, Zero J. Kim, S. Song

Korea University, Seoul, Korea

S. Choi, D. Gyun, B. Hong, M. Jo, H. Kim, Y. Kim, K.S. Lee, S.K. Park, Y. Roh

University of Seoul, Seoul, Korea

M. Choi, J.H. Kim, C. Park, I.C. Park, S. Park, G. Ryu

Sungkyunkwan University, Suwon, Korea

Y. Choi, Y.K. Choi, J. Goh, E. Kwon, B. Lee, J. Lee, H. Seo, I. Yu

Vilnius University, Vilnius, Lithuania

A. Juodagalvis

National Centre for Particle Physics, Universiti Malaya, Kuala Lumpur, Malaysia

J.R. Komaragiri

Centro de Investigacion y de Estudios Avanzados del IPN, Mexico City, Mexico

H. Castilla-Valdez, E. De La Cruz-Burelo, I. Heredia-de La Cruz ${ }^{31}$, R. Lopez-Fernandez, J. Martínez-Ortega, A. Sanchez-Hernandez, L.M. Villasenor-Cendejas

Universidad Iberoamericana, Mexico City, Mexico

S. Carrillo Moreno, F. Vazquez Valencia

Benemerita Universidad Autonoma de Puebla, Puebla, Mexico

H.A. Salazar Ibarguen

Universidad Autónoma de San Luis Potosí, San Luis Potosí, Mexico

E. Casimiro Linares, A. Morelos Pineda

University of Auckland, Auckland, New Zealand

D. Krofcheck

University of Canterbury, Christchurch, New Zealand

P.H. Butler, R. Doesburg, S. Reucroft

National Centre for Physics, Quaid-I-Azam University, Islamabad, Pakistan

M. Ahmad, M.I. Asghar, J. Butt, H.R. Hoorani, W.A. Khan, T. Khurshid, S. Qazi, M.A. Shah, M. Shoaib

National Centre for Nuclear Research, Swierk, Poland

H. Bialkowska, M. Bluj ${ }^{32}$, B. Boimska, T. Frueboes, M. Górski, M. Kazana, K. Nawrocki,

K. Romanowska-Rybinska, M. Szleper, G. Wrochna, P. Zalewski 
Institute of Experimental Physics, Faculty of Physics, University of Warsaw, Warsaw, Poland

G. Brona, K. Bunkowski, M. Cwiok, W. Dominik, K. Doroba, A. Kalinowski, M. Konecki, J. Krolikowski, M. Misiura, W. Wolszczak

Laboratório de Instrumentação e Física Experimental de Partículas, Lisboa, Portugal

P. Bargassa, C. Beirão Da Cruz E Silva, P. Faccioli, P.G. Ferreira Parracho, M. Gallinaro, F. Nguyen, J. Rodrigues Antunes, J. Seixas², J. Varela, P. Vischia

Joint Institute for Nuclear Research, Dubna, Russia

S. Afanasiev, P. Bunin, M. Gavrilenko, I. Golutvin, V. Karjavin, V. Konoplyanikov,

G. Kozlov, A. Lanev, A. Malakhov, V. Matveev ${ }^{33}$, P. Moisenz, V. Palichik, V. Perelygin,

M. Savina, S. Shmatov, N. Skatchkov, V. Smirnov, A. Zarubin

Petersburg Nuclear Physics Institute, Gatchina (St. Petersburg), Russia

V. Golovtsov, Y. Ivanov, V. Kim ${ }^{34}$, P. Levchenko, V. Murzin, V. Oreshkin, I. Smirnov, V. Sulimov, L. Uvarov, S. Vavilov, A. Vorobyev, An. Vorobyev

Institute for Nuclear Research, Moscow, Russia

Yu. Andreev, A. Dermenev, S. Gninenko, N. Golubev, M. Kirsanov, N. Krasnikov, A. Pashenkov, D. Tlisov, A. Toropin

Institute for Theoretical and Experimental Physics, Moscow, Russia

V. Epshteyn, V. Gavrilov, N. Lychkovskaya, V. Popov, G. Safronov, S. Semenov, A. Spiridonov, V. Stolin, E. Vlasov, A. Zhokin

P.N. Lebedev Physical Institute, Moscow, Russia

V. Andreev, M. Azarkin, I. Dremin, M. Kirakosyan, A. Leonidov, G. Mesyats, S.V. Rusakov, A. Vinogradov

Skobeltsyn Institute of Nuclear Physics, Lomonosov Moscow State University, Moscow, Russia

A. Belyaev, E. Boos, V. Bunichev, M. Dubinin ${ }^{7}$, L. Dudko, A. Gribushin, V. Klyukhin, I. Lokhtin, S. Obraztsov, M. Perfilov, S. Petrushanko, V. Savrin, A. Snigirev

State Research Center of Russian Federation, Institute for High Energy Physics, Protvino, Russia

I. Azhgirey, I. Bayshev, S. Bitioukov, V. Kachanov, A. Kalinin, D. Konstantinov, V. Krychkine, V. Petrov, R. Ryutin, A. Sobol, L. Tourtchanovitch, S. Troshin, N. Tyurin, A. Uzunian, A. Volkov

University of Belgrade, Faculty of Physics and Vinca Institute of Nuclear Sciences, Belgrade, Serbia

P. Adzic ${ }^{35}$, M. Djordjevic, M. Ekmedzic, J. Milosevic 
Centro de Investigaciones Energéticas Medioambientales y Tecnológicas (CIEMAT), Madrid, Spain

M. Aguilar-Benitez, J. Alcaraz Maestre, C. Battilana, E. Calvo, M. Cerrada, M. Chamizo Llatas², N. Colino, B. De La Cruz, A. Delgado Peris, D. Domínguez Vázquez, C. Fernandez Bedoya, J.P. Fernández Ramos, A. Ferrando, J. Flix, M.C. Fouz, P. Garcia-Abia, O. Gonzalez Lopez, S. Goy Lopez, J.M. Hernandez, M.I. Josa, G. Merino, E. Navarro De Martino, J. Puerta Pelayo, A. Quintario Olmeda, I. Redondo, L. Romero, M.S. Soares, C. Willmott

Universidad Autónoma de Madrid, Madrid, Spain

C. Albajar, J.F. de Trocóniz, M. Missiroli

\section{Universidad de Oviedo, Oviedo, Spain}

H. Brun, J. Cuevas, J. Fernandez Menendez, S. Folgueras, I. Gonzalez Caballero, L. Lloret Iglesias

Instituto de Física de Cantabria (IFCA), CSIC-Universidad de Cantabria, Santander, Spain

J.A. Brochero Cifuentes, I.J. Cabrillo, A. Calderon, J. Duarte Campderros, M. Fernandez, G. Gomez, J. Gonzalez Sanchez, A. Graziano, A. Lopez Virto, J. Marco, R. Marco, C. Martinez Rivero, F. Matorras, F.J. Munoz Sanchez, J. Piedra Gomez, T. Rodrigo, A.Y. Rodríguez-Marrero, A. Ruiz-Jimeno, L. Scodellaro, I. Vila, R. Vilar Cortabitarte

\section{CERN, European Organization for Nuclear Research, Geneva, Switzerland}

D. Abbaneo, E. Auffray, G. Auzinger, M. Bachtis, P. Baillon, A.H. Ball, D. Barney, J. Bendavid, L. Benhabib, J.F. Benitez, C. Bernet ${ }^{8}$, G. Bianchi, P. Bloch, A. Bocci, A. Bonato, O. Bondu, C. Botta, H. Breuker, T. Camporesi, G. Cerminara, T. Christiansen, J.A. Coarasa Perez, S. Colafranceschi ${ }^{36}$, M. D'Alfonso, D. d'Enterria, A. Dabrowski, A. David, F. De Guio, A. De Roeck, S. De Visscher, M. Dobson, N. Dupont-Sagorin, A. Elliott-Peisert, J. Eugster, G. Franzoni, W. Funk, M. Giffels, D. Gigi, K. Gill, D. Giordano, M. Girone, M. Giunta, F. Glege, R. Gomez-Reino Garrido, S. Gowdy, R. Guida, J. Hammer, M. Hansen, P. Harris, V. Innocente, P. Janot, E. Karavakis, K. Kousouris, K. Krajczar, P. Lecoq, C. Lourenço, N. Magini, L. Malgeri, M. Mannelli, L. Masetti, F. Meijers, S. Mersi, E. Meschi, F. Moortgat, M. Mulders, P. Musella, L. Orsini, E. Palencia Cortezon, E. Perez, L. Perrozzi, A. Petrilli, G. Petrucciani, A. Pfeiffer, M. Pierini, M. Pimiä, D. Piparo, M. Plagge, A. Racz, W. Reece, G. Rolandi' ${ }^{37}$, M. Rovere, H. Sakulin, F. Santanastasio, C. Schäfer, C. Schwick, S. Sekmen, A. Sharma, P. Siegrist, P. Silva, M. Simon, P. Sphicas ${ }^{38}$, D. Spiga, J. Steggemann, B. Stieger, M. Stoye, A. Tsirou, G.I. Veres ${ }^{20}$, J.R. Vlimant, H.K. Wöhri, W.D. Zeuner

\section{Paul Scherrer Institut, Villigen, Switzerland}

W. Bertl, K. Deiters, W. Erdmann, R. Horisberger, Q. Ingram, H.C. Kaestli, S. König, D. Kotlinski, U. Langenegger, D. Renker, T. Rohe 
Institute for Particle Physics, ETH Zurich, Zurich, Switzerland

F. Bachmair, L. Bäni, L. Bianchini, P. Bortignon, M.A. Buchmann, B. Casal, N. Chanon, A. Deisher, G. Dissertori, M. Dittmar, M. Donegà, M. Dünser, P. Eller, C. Grab, D. Hits, W. Lustermann, B. Mangano, A.C. Marini, P. Martinez Ruiz del Arbol, D. Meister, N. Mohr, C. Nägeli ${ }^{39}$, P. Nef, F. Nessi-Tedaldi, F. Pandolfi, L. Pape, F. Pauss, M. Peruzzi, M. Quittnat, F.J. Ronga, M. Rossini, A. Starodumov ${ }^{40}$, M. Takahashi, L. Tauscher ${ }^{\dagger}$, K. Theofilatos, D. Treille, R. Wallny, H.A. Weber

Universität Zürich, Zurich, Switzerland

C. Amsler ${ }^{41}$, M.F. Canelli, V. Chiochia, A. De Cosa, C. Favaro, A. Hinzmann, T. Hreus, M. Ivova Rikova, B. Kilminster, B. Millan Mejias, J. Ngadiuba, P. Robmann, H. Snoek, S. Taroni, M. Verzetti, Y. Yang

National Central University, Chung-Li, Taiwan

M. Cardaci, K.H. Chen, C. Ferro, C.M. Kuo, S.W. Li, W. Lin, Y.J. Lu, R. Volpe, S.S. Yu National Taiwan University (NTU), Taipei, Taiwan

P. Bartalini, P. Chang, Y.H. Chang, Y.W. Chang, Y. Chao, K.F. Chen, P.H. Chen,

C. Dietz, U. Grundler, W.-S. Hou, Y. Hsiung, K.Y. Kao, Y.J. Lei, Y.F. Liu, R.-S. Lu,

D. Majumder, E. Petrakou, X. Shi, J.G. Shiu, Y.M. Tzeng, M. Wang, R. Wilken

\section{Chulalongkorn University, Bangkok, Thailand}

B. Asavapibhop, N. Suwonjandee

\section{Cukurova University, Adana, Turkey}

A. Adiguzel, M.N. Bakirci ${ }^{42}$, S. Cerci ${ }^{43}$, C. Dozen, I. Dumanoglu, E. Eskut, S. Girgis, G. Gokbulut, E. Gurpinar, I. Hos, E.E. Kangal, A. Kayis Topaksu, G. Onengut ${ }^{44}$, K. Ozdemir, S. Ozturk ${ }^{42}$, A. Polatoz, K. Sogut ${ }^{45}$, D. Sunar Cerci ${ }^{43}$, B. Tali ${ }^{43}$, H. Topakli ${ }^{42}$, M. Vergili

Middle East Technical University, Physics Department, Ankara, Turkey

I.V. Akin, T. Aliev, B. Bilin, S. Bilmis, M. Deniz, H. Gamsizkan, A.M. Guler, G. Karapinar ${ }^{46}$, K. Ocalan, A. Ozpineci, M. Serin, R. Sever, U.E. Surat, M. Yalvac, M. Zeyrek

Bogazici University, Istanbul, Turkey

E. Gülmez, B. Isildak ${ }^{47}$, M. Kaya ${ }^{48}$, O. Kaya ${ }^{48}$, S. Ozkorucuklu ${ }^{49}$

Istanbul Technical University, Istanbul, Turkey

H. Bahtiyar ${ }^{50}$, E. Barlas, K. Cankocak, Y.O. Günaydin ${ }^{51}$, F.I. Vardarlı, M. Yücel

National Scientific Center, Kharkov Institute of Physics and Technology, Kharkov, Ukraine

L. Levchuk, P. Sorokin

University of Bristol, Bristol, United Kingdom

J.J. Brooke, E. Clement, D. Cussans, H. Flacher, R. Frazier, J. Goldstein, M. Grimes, G.P. Heath, H.F. Heath, J. Jacob, L. Kreczko, C. Lucas, Z. Meng, D.M. Newbold ${ }^{52}$, S. Paramesvaran, A. Poll, S. Senkin, V.J. Smith, T. Williams 


\section{Rutherford Appleton Laboratory, Didcot, United Kingdom}

K.W. Bell, A. Belyaev ${ }^{53}$, C. Brew, R.M. Brown, D.J.A. Cockerill, J.A. Coughlan, K. Harder, S. Harper, J. Ilic, E. Olaiya, D. Petyt, C.H. Shepherd-Themistocleous, A. Thea, I.R. Tomalin, W.J. Womersley, S.D. Worm

\section{Imperial College, London, United Kingdom}

M. Baber, R. Bainbridge, O. Buchmuller, D. Burton, D. Colling, N. Cripps, M. Cutajar, P. Dauncey, G. Davies, M. Della Negra, W. Ferguson, J. Fulcher, D. Futyan, A. Gilbert, A. Guneratne Bryer, G. Hall, Z. Hatherell, J. Hays, G. Iles, M. Jarvis, G. Karapostoli, M. Kenzie, R. Lane, R. Lucas ${ }^{52}$, L. Lyons, A.-M. Magnan, J. Marrouche, B. Mathias, R. Nandi, J. Nash, A. Nikitenko ${ }^{40}$, J. Pela, M. Pesaresi, K. Petridis, M. Pioppi ${ }^{54}$, D.M. Raymond, S. Rogerson, A. Rose, C. Seez, P. Sharp ${ }^{\dagger}$, A. Sparrow, A. Tapper, M. Vazquez Acosta, T. Virdee, S. Wakefield, N. Wardle

\section{Brunel University, Uxbridge, United Kingdom}

J.E. Cole, P.R. Hobson, A. Khan, P. Kyberd, D. Leggat, D. Leslie, W. Martin, I.D. Reid, P. Symonds, L. Teodorescu, M. Turner

Baylor University, Waco, U.S.A.

J. Dittmann, K. Hatakeyama, A. Kasmi, H. Liu, T. Scarborough

The University of Alabama, Tuscaloosa, U.S.A.

O. Charaf, S.I. Cooper, C. Henderson, P. Rumerio

Boston University, Boston, U.S.A.

A. Avetisyan, T. Bose, C. Fantasia, A. Heister, P. Lawson, D. Lazic, J. Rohlf, D. Sperka, J. St. John, L. Sulak

Brown University, Providence, U.S.A.

J. Alimena, S. Bhattacharya, G. Christopher, D. Cutts, Z. Demiragli, A. Ferapontov, A. Garabedian, U. Heintz, S. Jabeen, G. Kukartsev, E. Laird, G. Landsberg, M. Luk, M. Narain, M. Segala, T. Sinthuprasith, T. Speer, J. Swanson

University of California, Davis, Davis, U.S.A.

R. Breedon, G. Breto, M. Calderon De La Barca Sanchez, S. Chauhan, M. Chertok, J. Conway, R. Conway, P.T. Cox, R. Erbacher, M. Gardner, W. Ko, A. Kopecky, R. Lander, T. Miceli, D. Pellett, J. Pilot, F. Ricci-Tam, B. Rutherford, M. Searle, S. Shalhout, J. Smith, M. Squires, M. Tripathi, S. Wilbur, R. Yohay

University of California, Los Angeles, U.S.A.

V. Andreev, D. Cline, R. Cousins, S. Erhan, P. Everaerts, C. Farrell, M. Felcini, J. Hauser, M. Ignatenko, C. Jarvis, G. Rakness, P. Schlein ${ }^{\dagger}$, E. Takasugi, V. Valuev, M. Weber

University of California, Riverside, Riverside, U.S.A.

J. Babb, R. Clare, J. Ellison, J.W. Gary, G. Hanson, J. Heilman, P. Jandir, F. Lacroix, H. Liu, O.R. Long, A. Luthra, M. Malberti, H. Nguyen, A. Shrinivas, J. Sturdy, S. Sumowidagdo, S. Wimpenny 
University of California, San Diego, La Jolla, U.S.A.

W. Andrews, J.G. Branson, G.B. Cerati, S. Cittolin, R.T. D’Agnolo, D. Evans, A. Holzner, R. Kelley, D. Kovalskyi, M. Lebourgeois, J. Letts, I. Macneill, S. Padhi, C. Palmer, M. Pieri, M. Sani, V. Sharma, S. Simon, E. Sudano, M. Tadel, Y. Tu, A. Vartak, S. Wasserbaech ${ }^{55}$, F. Würthwein, A. Yagil, J. Yoo

\section{University of California, Santa Barbara, Santa Barbara, U.S.A.}

D. Barge, C. Campagnari, T. Danielson, K. Flowers, P. Geffert, C. George, F. Golf, J. Incandela, C. Justus, R. Magaña Villalba, N. Mccoll, V. Pavlunin, J. Richman, R. Rossin, D. Stuart, W. To, C. West

\section{California Institute of Technology, Pasadena, U.S.A.}

A. Apresyan, A. Bornheim, J. Bunn, Y. Chen, E. Di Marco, J. Duarte, D. Kcira, A. Mott, H.B. Newman, C. Pena, C. Rogan, M. Spiropulu, V. Timciuc, R. Wilkinson, S. Xie, R.Y. Zhu

\section{Carnegie Mellon University, Pittsburgh, U.S.A.}

V. Azzolini, A. Calamba, R. Carroll, T. Ferguson, Y. Iiyama, D.W. Jang, M. Paulini, J. Russ, H. Vogel, I. Vorobiev

University of Colorado at Boulder, Boulder, U.S.A.

J.P. Cumalat, B.R. Drell, W.T. Ford, A. Gaz, E. Luiggi Lopez, U. Nauenberg, J.G. Smith, K. Stenson, K.A. Ulmer, S.R. Wagner

Cornell University, Ithaca, U.S.A.

J. Alexander, A. Chatterjee, N. Eggert, L.K. Gibbons, W. Hopkins, A. Khukhunaishvili, B. Kreis, N. Mirman, G. Nicolas Kaufman, J.R. Patterson, A. Ryd, E. Salvati, W. Sun, W.D. Teo, J. Thom, J. Thompson, J. Tucker, Y. Weng, L. Winstrom, P. Wittich

Fairfield University, Fairfield, U.S.A.

D. Winn

Fermi National Accelerator Laboratory, Batavia, U.S.A.

S. Abdullin, M. Albrow, J. Anderson, G. Apollinari, L.A.T. Bauerdick, A. Beretvas, J. Berryhill, P.C. Bhat, K. Burkett, J.N. Butler, V. Chetluru, H.W.K. Cheung, F. Chlebana, S. Cihangir, V.D. Elvira, I. Fisk, J. Freeman, Y. Gao, E. Gottschalk, L. Gray, D. Green, S. Grünendahl, O. Gutsche, D. Hare, R.M. Harris, J. Hirschauer, B. Hooberman, S. Jindariani, M. Johnson, U. Joshi, K. Kaadze, B. Klima, S. Kwan, J. Linacre, D. Lincoln, R. Lipton, J. Lykken, K. Maeshima, J.M. Marraffino, V.I. Martinez Outschoorn, S. Maruyama, D. Mason, P. McBride, K. Mishra, S. Mrenna, Y. Musienko ${ }^{33}$, S. Nahn, C. Newman-Holmes, V. O'Dell, O. Prokofyev, N. Ratnikova, E. Sexton-Kennedy, S. Sharma, W.J. Spalding, L. Spiegel, L. Taylor, S. Tkaczyk, N.V. Tran, L. Uplegger, E.W. Vaandering, R. Vidal, A. Whitbeck, J. Whitmore, W. Wu, F. Yang, J.C. Yun

University of Florida, Gainesville, U.S.A.

D. Acosta, P. Avery, D. Bourilkov, T. Cheng, S. Das, M. De Gruttola, G.P. Di Giovanni, D. Dobur, R.D. Field, M. Fisher, Y. Fu, I.K. Furic, J. Hugon, B. Kim, J. Konigsberg, 
A. Korytov, A. Kropivnitskaya, T. Kypreos, J.F. Low, K. Matchev, P. Milenovic ${ }^{56}$, G. Mitselmakher, L. Muniz, A. Rinkevicius, L. Shchutska, N. Skhirtladze, M. Snowball, J. Yelton, M. Zakaria

Florida International University, Miami, U.S.A.

V. Gaultney, S. Hewamanage, S. Linn, P. Markowitz, G. Martinez, J.L. Rodriguez

Florida State University, Tallahassee, U.S.A.

T. Adams, A. Askew, J. Bochenek, J. Chen, B. Diamond, J. Haas, S. Hagopian, V. Hagopian, K.F. Johnson, H. Prosper, V. Veeraraghavan, M. Weinberg

Florida Institute of Technology, Melbourne, U.S.A.

M.M. Baarmand, B. Dorney, M. Hohlmann, H. Kalakhety, F. Yumiceva

University of Illinois at Chicago (UIC), Chicago, U.S.A.

M.R. Adams, L. Apanasevich, V.E. Bazterra, R.R. Betts, I. Bucinskaite, R. Cavanaugh, O. Evdokimov, L. Gauthier, C.E. Gerber, D.J. Hofman, S. Khalatyan, P. Kurt, D.H. Moon, C. O'Brien, C. Silkworth, P. Turner, N. Varelas

The University of Iowa, Iowa City, U.S.A.

U. Akgun, E.A. Albayrak ${ }^{50}$, B. Bilki ${ }^{57}$, W. Clarida, K. Dilsiz, F. Duru, M. Haytmyradov, J.-P. Merlo, H. Mermerkaya ${ }^{58}$, A. Mestvirishvili, A. Moeller, J. Nachtman, H. Ogul, Y. Onel, F. Ozok ${ }^{50}$, S. Sen, P. Tan, E. Tiras, J. Wetzel, T. Yetkin ${ }^{59}$, K. Yi

Johns Hopkins University, Baltimore, U.S.A.

B.A. Barnett, B. Blumenfeld, S. Bolognesi, D. Fehling, A.V. Gritsan, P. Maksimovic, C. Martin, M. Swartz

The University of Kansas, Lawrence, U.S.A.

P. Baringer, A. Bean, G. Benelli, R.P. Kenny III, M. Murray, D. Noonan, S. Sanders, J. Sekaric, R. Stringer, Q. Wang, J.S. Wood

Kansas State University, Manhattan, U.S.A.

A.F. Barfuss, I. Chakaberia, A. Ivanov, S. Khalil, M. Makouski, Y. Maravin, L.K. Saini, S. Shrestha, I. Svintradze

Lawrence Livermore National Laboratory, Livermore, U.S.A.

J. Gronberg, D. Lange, F. Rebassoo, D. Wright

University of Maryland, College Park, U.S.A.

A. Baden, B. Calvert, S.C. Eno, J.A. Gomez, N.J. Hadley, R.G. Kellogg, T. Kolberg, Y. Lu, M. Marionneau, A.C. Mignerey, K. Pedro, A. Skuja, J. Temple, M.B. Tonjes, S.C. Tonwar Massachusetts Institute of Technology, Cambridge, U.S.A.

A. Apyan, R. Barbieri, G. Bauer, W. Busza, I.A. Cali, M. Chan, L. Di Matteo, V. Dutta, G. Gomez Ceballos, M. Goncharov, D. Gulhan, M. Klute, Y.S. Lai, Y.-J. Lee, A. Levin, P.D. Luckey, T. Ma, C. Paus, D. Ralph, C. Roland, G. Roland, G.S.F. Stephans, F. Stöckli, K. Sumorok, D. Velicanu, J. Veverka, B. Wyslouch, M. Yang, A.S. Yoon, M. Zanetti, V. Zhukova 
University of Minnesota, Minneapolis, U.S.A.

B. Dahmes, A. De Benedetti, A. Gude, S.C. Kao, K. Klapoetke, Y. Kubota, J. Mans,

N. Pastika, R. Rusack, A. Singovsky, N. Tambe, J. Turkewitz

University of Mississippi, Oxford, U.S.A.

J.G. Acosta, L.M. Cremaldi, R. Kroeger, S. Oliveros, L. Perera, R. Rahmat, D.A. Sanders,

D. Summers

University of Nebraska-Lincoln, Lincoln, U.S.A.

E. Avdeeva, K. Bloom, S. Bose, D.R. Claes, A. Dominguez, R. Gonzalez Suarez, J. Keller,

D. Knowlton, I. Kravchenko, J. Lazo-Flores, S. Malik, F. Meier, G.R. Snow

State University of New York at Buffalo, Buffalo, U.S.A.

J. Dolen, A. Godshalk, I. Iashvili, S. Jain, A. Kharchilava, A. Kumar, S. Rappoccio

Northeastern University, Boston, U.S.A.

G. Alverson, E. Barberis, D. Baumgartel, M. Chasco, J. Haley, A. Massironi, D. Nash,

T. Orimoto, D. Trocino, D. Wood, J. Zhang

Northwestern University, Evanston, U.S.A.

A. Anastassov, K.A. Hahn, A. Kubik, L. Lusito, N. Mucia, N. Odell, B. Pollack, A. Pozdnyakov, M. Schmitt, S. Stoynev, K. Sung, M. Velasco, S. Won

University of Notre Dame, Notre Dame, U.S.A.

D. Berry, A. Brinkerhoff, K.M. Chan, A. Drozdetskiy, M. Hildreth, C. Jessop, D.J. Karmgard, N. Kellams, J. Kolb, K. Lannon, W. Luo, S. Lynch, N. Marinelli, D.M. Morse, T. Pearson, M. Planer, R. Ruchti, J. Slaunwhite, N. Valls, M. Wayne, M. Wolf, A. Woodard

\section{The Ohio State University, Columbus, U.S.A.}

L. Antonelli, B. Bylsma, L.S. Durkin, S. Flowers, C. Hill, R. Hughes, K. Kotov, T.Y. Ling, D. Puigh, M. Rodenburg, G. Smith, C. Vuosalo, B.L. Winer, H. Wolfe, H.W. Wulsin

\section{Princeton University, Princeton, U.S.A.}

E. Berry, P. Elmer, V. Halyo, P. Hebda, J. Hegeman, A. Hunt, P. Jindal, S.A. Koay, P. Lujan, D. Marlow, T. Medvedeva, M. Mooney, J. Olsen, P. Piroué, X. Quan, A. Raval, H. Saka, D. Stickland, C. Tully, J.S. Werner, S.C. Zenz, A. Zuranski

University of Puerto Rico, Mayaguez, U.S.A.

E. Brownson, A. Lopez, H. Mendez, J.E. Ramirez Vargas

Purdue University, West Lafayette, U.S.A.

E. Alagoz, D. Benedetti, G. Bolla, D. Bortoletto, M. De Mattia, A. Everett, Z. Hu, M.K. Jha, M. Jones, K. Jung, M. Kress, N. Leonardo, D. Lopes Pegna, V. Maroussov, P. Merkel, D.H. Miller, N. Neumeister, B.C. Radburn-Smith, I. Shipsey, D. Silvers, A. Svyatkovskiy, F. Wang, W. Xie, L. Xu, H.D. Yoo, J. Zablocki, Y. Zheng

Purdue University Calumet, Hammond, U.S.A.

N. Parashar 
Rice University, Houston, U.S.A.

A. Adair, B. Akgun, K.M. Ecklund, F.J.M. Geurts, W. Li, B. Michlin, B.P. Padley, R. Redjimi, J. Roberts, J. Zabel

University of Rochester, Rochester, U.S.A.

B. Betchart, A. Bodek, R. Covarelli, P. de Barbaro, R. Demina, Y. Eshaq, T. Ferbel, A. Garcia-Bellido, P. Goldenzweig, J. Han, A. Harel, D.C. Miner, G. Petrillo, D. Vishnevskiy, M. Zielinski

\section{The Rockefeller University, New York, U.S.A.}

A. Bhatti, R. Ciesielski, L. Demortier, K. Goulianos, G. Lungu, S. Malik, C. Mesropian

Rutgers, The State University of New Jersey, Piscataway, U.S.A.

S. Arora, A. Barker, J.P. Chou, C. Contreras-Campana, E. Contreras-Campana, D. Duggan, D. Ferencek, Y. Gershtein, R. Gray, E. Halkiadakis, D. Hidas, A. Lath, S. Panwalkar, M. Park, R. Patel, V. Rekovic, J. Robles, S. Salur, S. Schnetzer, C. Seitz, S. Somalwar, R. Stone, S. Thomas, P. Thomassen, M. Walker

University of Tennessee, Knoxville, U.S.A.

K. Rose, S. Spanier, Z.C. Yang, A. York

Texas A\&M University, College Station, U.S.A.

O. Bouhali ${ }^{60}$, R. Eusebi, W. Flanagan, J. Gilmore, T. Kamon ${ }^{61}$, V. Khotilovich, V. Krutelyov, R. Montalvo, I. Osipenkov, Y. Pakhotin, A. Perloff, J. Roe, A. Safonov, T. Sakuma, I. Suarez, A. Tatarinov, D. Toback

Texas Tech University, Lubbock, U.S.A.

N. Akchurin, C. Cowden, J. Damgov, C. Dragoiu, P.R. Dudero, J. Faulkner, K. Kovitanggoon, S. Kunori, S.W. Lee, T. Libeiro, I. Volobouev

Vanderbilt University, Nashville, U.S.A.

E. Appelt, A.G. Delannoy, S. Greene, A. Gurrola, W. Johns, C. Maguire, Y. Mao, A. Melo, M. Sharma, P. Sheldon, B. Snook, S. Tuo, J. Velkovska

University of Virginia, Charlottesville, U.S.A.

M.W. Arenton, S. Boutle, B. Cox, B. Francis, J. Goodell, R. Hirosky, A. Ledovskoy, C. Lin, C. Neu, J. Wood

Wayne State University, Detroit, U.S.A.

S. Gollapinni, R. Harr, P.E. Karchin, C. Kottachchi Kankanamge Don, P. Lamichhane

University of Wisconsin, Madison, U.S.A.

D.A. Belknap, L. Borrello, D. Carlsmith, M. Cepeda, S. Dasu, S. Duric, E. Friis, M. Grothe, R. Hall-Wilton, M. Herndon, A. Hervé, P. Klabbers, J. Klukas, A. Lanaro, A. Levine, R. Loveless, A. Mohapatra, I. Ojalvo, T. Perry, G.A. Pierro, G. Polese, I. Ross, A. Sakharov, T. Sarangi, A. Savin, W.H. Smith, N. Woods 
$\dagger$ : Deceased

1: Also at Vienna University of Technology, Vienna, Austria

2: Also at CERN, European Organization for Nuclear Research, Geneva, Switzerland

3: Also at Institut Pluridisciplinaire Hubert Curien, Université de Strasbourg,

Université de Haute Alsace Mulhouse, CNRS/IN2P3, Strasbourg, France

4: Also at National Institute of Chemical Physics and Biophysics, Tallinn, Estonia

5: Also at Skobeltsyn Institute of Nuclear Physics, Lomonosov Moscow State University, Moscow, Russia

6: Also at Universidade Estadual de Campinas, Campinas, Brazil

7: Also at California Institute of Technology, Pasadena, U.S.A.

8: Also at Laboratoire Leprince-Ringuet, Ecole Polytechnique, IN2P3-CNRS, Palaiseau, France

9: Also at Zewail City of Science and Technology, Zewail, Egypt

10: Also at Suez Canal University, Suez, Egypt

11: Also at British University in Egypt, Cairo, Egypt

12: Also at Cairo University, Cairo, Egypt

13: Also at Fayoum University, El-Fayoum, Egypt

14: Now at Ain Shams University, Cairo, Egypt

15: Also at Université de Haute Alsace, Mulhouse, France

16: Also at Joint Institute for Nuclear Research, Dubna, Russia

17: Also at Brandenburg University of Technology, Cottbus, Germany

18: Also at The University of Kansas, Lawrence, U.S.A.

19: Also at Institute of Nuclear Research ATOMKI, Debrecen, Hungary

20: Also at Eötvös Loránd University, Budapest, Hungary

21: Also at Tata Institute of Fundamental Research - HECR, Mumbai, India

22: Now at King Abdulaziz University, Jeddah, Saudi Arabia

23: Also at University of Visva-Bharati, Santiniketan, India

24: Also at University of Ruhuna, Matara, Sri Lanka

25: Also at Isfahan University of Technology, Isfahan, Iran

26: Also at Sharif University of Technology, Tehran, Iran

27: Also at Plasma Physics Research Center, Science and Research Branch, Islamic Azad University, Tehran, Iran

28: Also at Università degli Studi di Siena, Siena, Italy

29: Also at Centre National de la Recherche Scientifique (CNRS) - IN2P3, Paris, France

30: Also at Purdue University, West Lafayette, U.S.A.

31: Also at Universidad Michoacana de San Nicolas de Hidalgo, Morelia, Mexico

32: Also at National Centre for Nuclear Research, Swierk, Poland

33: Also at Institute for Nuclear Research, Moscow, Russia

34: Also at St. Petersburg State Polytechnical University, St. Petersburg, Russia

35: Also at Faculty of Physics, University of Belgrade, Belgrade, Serbia

36: Also at Facoltà Ingegneria, Università di Roma, Roma, Italy

37: Also at Scuola Normale e Sezione dell'INFN, Pisa, Italy

38: Also at University of Athens, Athens, Greece

39: Also at Paul Scherrer Institut, Villigen, Switzerland

40: Also at Institute for Theoretical and Experimental Physics, Moscow, Russia

41: Also at Albert Einstein Center for Fundamental Physics, Bern, Switzerland

42: Also at Gaziosmanpasa University, Tokat, Turkey

43: Also at Adiyaman University, Adiyaman, Turkey

44: Also at Cag University, Mersin, Turkey 
45: Also at Mersin University, Mersin, Turkey

46: Also at Izmir Institute of Technology, Izmir, Turkey

47: Also at Ozyegin University, Istanbul, Turkey

48: Also at Kafkas University, Kars, Turkey

49: Also at Istanbul University, Faculty of Science, Istanbul, Turkey

50: Also at Mimar Sinan University, Istanbul, Istanbul, Turkey

51: Also at Kahramanmaras Sütcü Imam University, Kahramanmaras, Turkey

52: Also at Rutherford Appleton Laboratory, Didcot, United Kingdom

53: Also at School of Physics and Astronomy, University of Southampton, Southampton, United Kingdom

54: Also at INFN Sezione di Perugia; Università di Perugia, Perugia, Italy

55: Also at Utah Valley University, Orem, U.S.A.

56: Also at University of Belgrade, Faculty of Physics and Vinca Institute of Nuclear Sciences, Belgrade, Serbia

57: Also at Argonne National Laboratory, Argonne, U.S.A.

58: Also at Erzincan University, Erzincan, Turkey

59: Also at Yildiz Technical University, Istanbul, Turkey

60: Also at Texas A\&M University at Qatar, Doha, Qatar

61: Also at Kyungpook National University, Daegu, Korea 